\title{
Surface defects and elliptic quantum groups
}

\author{
Junya Yagi \\ Faculty of Physics, University of Warsaw, \\ ul. Pasteura 5, 02-093 Warsaw, Poland \\ E-mail: junya.yagi@fuw.edu.pl
}

ABSTRACT: A brane construction of an integrable lattice model is proposed. The model is composed of Belavin's R-matrix, Felder's dynamical R-matrix, the Bazhanov-SergeevDerkachov-Spiridonov R-operator and some intertwining operators. This construction implies that a family of surface defects act on supersymmetric indices of four-dimensional $\mathcal{N}=1$ supersymmetric field theories as transfer matrices related to elliptic quantum groups.

KEYwords: Lattice Integrable Models, p-branes, Quantum Groups, Supersymmetric Gauge Theory

ARXIV EPRINT: 1701.05562 


\section{Contents}

1 Introduction $\quad 1$

2 Integrable lattice models of elliptic type 4

2.1 Belavin model 5

2.2 Felder's dynamical R-matrix $\quad 7$

2.3 Vertex-face correspondence and Jimbo-Miwa-Okado model 9

2.4 Bazhanov-Sergeev model 9

3 Unification $\quad 13$

$\begin{array}{lll}3.1 & \text { Intertwining operators } & 13\end{array}$

$\begin{array}{lll}3.2 & \text { Yang-Baxter equations with one dashed line } & 14\end{array}$

$\begin{array}{lll}3.3 & \text { Yang-Baxter equations with two dashed lines } & 17\end{array}$

$\begin{array}{lll}3.4 & \text { L-operators and elliptic quantum groups } & 18\end{array}$

4 Surface defects and elliptic quantum groups $\quad 20$

$\begin{array}{lll}4.1 & \text { Brane construction } & 21\end{array}$

$4.2 \mathcal{N}=1$ supersymmetric quiver gauge theories and surface defects 23

4.3 Fusion procedure and exterior powers of the vector representation 25

\section{Introduction}

The aim of this paper is twofold. It is an attempt to offer a fresh perspective on integrable lattice models of elliptic type, such as Baxter's eight-vertex model [1, 2], by embedding them into string theory. At the same time, it seeks to provide a new approach to the study of four-dimensional supersymmetric field theories by connecting their surface operators to those models.

More specifically, I propose a brane construction of an integrable lattice model that is composed of Belavin's R-matrix [3], Felder's dynamical R-matrix [4, 5], the BazhanovSergeev-Derkachov-Spiridonov (BSDS) R-operator [6-8] and intertwining operators between the first two R-matrices [9, 10]. This construction allows us to map a family of surface defects in $\mathcal{N}=1$ supersymmetric field theories to transfer matrices related to Felder's elliptic quantum groups.

The proposal builds on a recent development [11] in the correspondence between $\mathcal{N}=1$ supersymmetric field theories and integrable lattice models [12-15]. Therefore, let me first review the relevant results.

One side of the correspondence is a class of theories realized by certain configurations of 5-branes in string theory, referred to as brane tilings [16, 17]. A theory in this class 


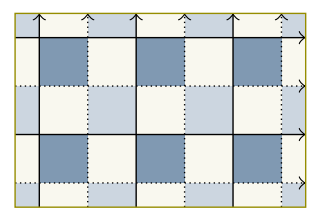

(a)

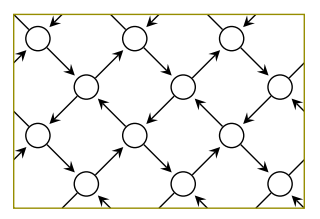

(b)

Figure 1. (a) A periodic lattice colored in a checkerboard-like pattern. (b) The quiver associated with the lattice. Each node is an $\mathrm{SU}(N)$ gauge group and each arrow is a matter multiplet.

has multiple $\mathrm{SU}(N)$ gauge and flavor groups as well as matter fields transforming in bifundamental representations under these groups, where $N$ is an integer fixed by the brane configuration. It is an example of a quiver gauge theory; its field content can be encapsulated in a planar quiver diagram. The quiver itself is specified by a square lattice whose faces are colored in a checkerboard-like pattern, which encodes the topology of the 5-branes interwoven in a ten-dimensional spacetime. The lattice has two kinds of "black" faces, either light shaded or dark shaded, and two shaded faces sharing a vertex is required to be of different kinds. Figure 1 shows an example of such a lattice and the associated quiver.

The other side of the correspondence is the Bazhanov-Sergeev model [6, 7], defined on the same tricolor checkerboard lattice. To each unshaded face is assigned a continuous spin variable that takes values in a maximal torus of $\mathrm{SU}(N)$. The Boltzmann weight, or R-operator, of the model is an integral operator involving elliptic gamma functions. It solves the Yang-Baxter equation, ensuring that the model is integrable.

In $[12,13]$, it was discovered that the supersymmetric index of the gauge theory, formulated on the three-sphere $S^{3}$, coincides with the partition function of the lattice model. Under this correspondence, the gauge and flavor groups are mapped to the spin sites, while the matters are interpreted as interactions between spins located at different sites. The Yang-Baxter equation translates on the gauge theory side to the invariance of the index under Seiberg duality [18], which relates two theories describing the same infrared physics.

As elucidated in [14], what underlies this correspondence is the structure of a twodimensional topological quantum field theory (TQFT), equipped with line operators that are localized in an extra dimension. From the fact that the supersymmetric index is invariant under continuous deformations of the checkerboard lattice, one deduces that it is captured by a correlation function of line operators in a TQFT. A general argument in open/closed TQFTs [15] then shows that the correlation function equals the partition function of a lattice model, the Bazhanov-Sergeev model in this case. Finally, the TQFT has a hidden extra dimension that emerges via string dualities, and its existence implies the integrability of the model $[19,20]$.

Things get more interesting when we introduce D3-branes that end on the 5-branes. With their configurations chosen appropriately, these additional branes create in the gauge theory surface defects preserving half of the $\mathcal{N}=1$ supersymmetry. In the checkerboard lattice they are supported along curves, which we represent by dashed lines as in figure 2(a). The same reasoning as above, applied to this situation, leads to the conclusion [11] that 


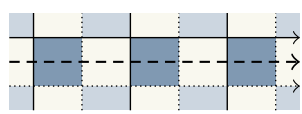

(a)

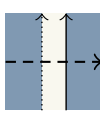

(b)

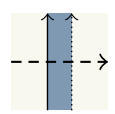

(c)

Figure 2. (a) A dashed line representing a D3-brane. It acts on the Bazhanov-Sergeev model as a transfer matrix. (b) The L-operator from which the transfer matrix is constructed. It is also an L-operator for the Belavin model. (c) An L-operator for Felder's R-matrix. It is obtained from the L-operator for the Belavin model by an interchange of the left and right halves.

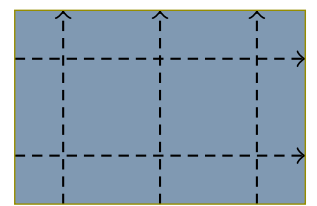

(a)

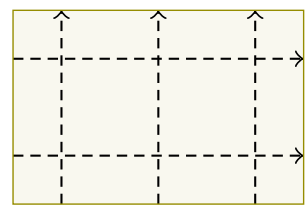

(b)

Figure 3. (a) A lattice formed by D3-branes in a shaded background supports the Belavin model. (b) The same lattice in an unshaded background gives rise to the Jimbo-Miwa-Okado model.

they act on the lattice model as transfer matrices that consist of so-called L-operators. An example of an L-operator is depicted in figure 2(b).

In [11], the relevant L-operator was identified for $N=2$, based on the analysis carried out in [8] and independent gauge theory computations. It was found that this L-operator is essentially Sklyanin's L-operator [21], and satisfies a defining "RLL relation" not only with the BSDS R-operator but also with Baxter's R-matrix for the eight-vertex model - a property that uplifts the well-known relation [22] between the chiral Potts model and the six-vertex model to the elliptic level. This fact strongly suggests that for $N=2$, the eightvertex model arises when D3-branes form a lattice in a shaded background, as illustrated in figure $3(\mathrm{a})$.

In this paper, the above result is generalized in a few interrelated directions.

First of all, the model realized on the lattice of D3-branes is identified in the general

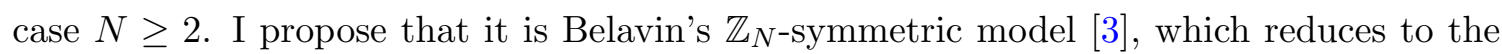
eight-vertex model when $N=2$. This proposal is backed up by the observation that there exists a simultaneous L-operator for the Belavin model and the Bazhanov-Sergeev model, and it factorizes [23-25] into a pair of intertwining operators. The factorization structure is manifest in the graphical representation of the L-operator, in which a single intertwining operator corresponds to either the left or right half.

A question then arises as to what the same lattice gives rise to when it is placed in an unshaded background, as in figure 3(b). The answer should be the model defined by Felder's dynamical R-matrix for the elliptic quantum group associated with $\mathfrak{s l}_{N},{ }^{1}$ which is the unrestricted $A_{N-1}^{(1)}$ model of Jimbo, Miwa and Okado [10, 26, 27]. One of the reasons is that an interchange of the pair of intertwining operators turns the L-operator for the Belavin model into that for Felder's R-matrix. The latter L-operator is graphically represented as in figure 2(c), and defines a representation of the elliptic quantum group.

\footnotetext{
${ }^{1}$ The same conclusion was reached by Kevin Costello through consideration of a different physical setup.
} 
The brane construction therefore unifies three integrable lattice models, namely the Belavin, Jimbo-Miwa-Okado and Bazhanov-Sergeev models, with the help of intertwining operators. The vertex-face correspondence $[9,10]$ relating Belavin's and Felder's R-matrices can be understood as Yang-Baxter equations in this unified model, and as such, admits an interpretation in terms of brane movements. The intertwining operators are determined from these and other Yang-Baxter equations, and shown to lead to transfer matrices that agree with known results [11, 28] about surface defects in class- $\mathcal{S}_{k}$ theories [29].

Lastly, the correspondence with transfer matrices is extended to a family of surface defects labeled by the irreducible finite-dimensional representations of $\mathrm{SU}(N)$. Such a family is constructed with a little more elaborate brane configurations than the one described above, which can only handle the vector representation. This construction is identified with a method to generate arbitrary irreducible representations on the lattice model side, called the fusion procedure [30, 31]. The L-operators in the exterior powers of the vector representation are especially interesting since their traces produce [32] mutually commuting difference operators known as the Ruijsenaars operators [33]. These traces indeed match the difference operators that represent the corresponding surface defects acting on the indices of $\mathcal{N}=2$ supersymmetric field theories [34].

The paper is organized as follows. In section 2, we review the Belavin model, Felder's dynamical R-matrix and the Jimbo-Miwa-Okado model, and the Bazhanov-Sergeev model. In section 3, we construct an integrable lattice model that unifies the three models introduced in section 2, and explain how L-operators constructed from the intertwining operators provide representations of elliptic quantum groups. In section 4 , we discuss the brane construction and the correspondence between surface defects and transfer matrices, and check the proposal of the paper against gauge theory results.

\section{Integrable lattice models of elliptic type}

In this section we review the three integrable lattice models of elliptic type relevant to us: the Belavin model, the Jimbo-Miwa-Okado model defined by Felder's dynamical R-matrix, and the Bazhanov-Sergeev model. First we set up notation.

Fix an integer $N \geq 2$ and let $\left\{e_{1}, \ldots, e_{N}\right\}$ be the standard basis of $\mathbb{C}^{N}$. The Cartan subalgebra of $\mathfrak{s l}_{N}$ is $\mathfrak{h}=\left\{\operatorname{diag}\left(\lambda_{1}, \ldots, \lambda_{N}\right) \mid \sum_{i=1}^{N} \lambda_{i}=0\right\}$, the space of diagonal traceless complex $N \times N$ matrices. We make the identification $\mathfrak{h}^{*} \cong \mathfrak{h} \cong\left\{\lambda \in \mathbb{C}^{N} \mid \sum_{i=1}^{N} \lambda_{i}=0\right\}$, where the first isomorphism is induced by the nondegenerate bilinear form $(\lambda, \mu)=\operatorname{Tr}(\lambda \mu)$ on $\mathfrak{h}$. The vector representation $V=\mathbb{C}^{N}$ of $\mathfrak{s l}_{N}$ has the weight decomposition $V=$ $\bigoplus_{i=1}^{N} V\left[\omega_{i}\right]$, with $\omega_{i}=e_{i}-\sum_{j=1}^{N} e_{j} / N$ and $V\left[\omega_{i}\right]=\mathbb{C} e_{i}$.

Setting $z_{i}=e^{2 \pi \mathrm{i} \lambda_{i}}$, we obtain an $N$-tuple $\left(z_{1}, \ldots, z_{N}\right)$ of complex variables obeying the constraint $\prod_{i=1}^{N} z_{i}=1$. These variables parametrize a maximal torus of $\operatorname{SL}(N)$. We denote by $\mathbb{V}$ the space of meromorphic functions on the maximal torus that are invariant under the action of the Weyl group, i.e., the space of symmetric meromorphic functions of $\left(z_{1}, \ldots, z_{N}\right)$. Elements of the tensor product $\mathbb{V}^{\otimes n}$ may be thought of as meromorphic functions of $n$ sets of $N$ variables, symmetric with respect to each set. We extend $\mathbb{V}^{\otimes n}$ to mean the space of all such functions. 
Furthermore, fix complex parameters $\tau, \gamma$ with $\operatorname{Im} \tau, \operatorname{Im} \gamma>0$, and set $p=e^{2 \pi \mathrm{i} \tau}$ and $q=e^{2 \pi \mathrm{i} \gamma}$. The theta function with characteristics is defined by

$$
\theta\left[\begin{array}{l}
a \\
b
\end{array}\right](u \mid \tau)=\sum_{n=-\infty}^{\infty} e^{\pi \mathrm{i}(n+a)^{2} \tau+2 \pi \mathrm{i}(n+a)(u+b)} .
$$

We will need Jacobi's first theta function and the elliptic gamma function:

$$
\theta_{1}(u \mid \tau)=-\theta\left[\begin{array}{l}
1 / 2 \\
1 / 2
\end{array}\right](u \mid \tau), \quad \Gamma(z ; p, q)=\prod_{m, n=0}^{\infty} \frac{1-p^{m+1} q^{n+1} / z}{1-p^{m} q^{n} z} .
$$

They satisfy $\theta_{1}(u \mid \tau)=-\theta_{1}(-u \mid \tau)$ and $\Gamma(z ; p, q)=1 / \Gamma(p q / z ; p, q)$. We also introduce

$$
\theta^{(j)}(u \mid \tau, N)=\theta\left[\begin{array}{c}
1 / 2-j / N \\
1 / 2
\end{array}\right](u \mid N \tau), \quad \theta(z ; p)=(z ; p)_{\infty}(p / z ; p)_{\infty},
$$

where we have used the $q$-Pochhammer symbol $(z ; q)_{n}=\prod_{k=0}^{n-1}\left(1-q^{k} z\right)$. The modified theta function $\theta(z ; p)$ is related to $\theta_{1}(u \mid \tau)$ by $\theta_{1}(u \mid \tau)=\mathrm{i} p^{1 / 8}(p ; p)_{\infty} e^{-\pi \mathrm{i} u} \theta\left(e^{2 \pi \mathrm{i} u} ; p\right)$ and satisfies $\theta(z ; p)=\theta(p / z ; p)=-z \theta(1 / z ; p)$. Moreover, we have $\Gamma(q z ; p, q)=\theta(z ; p) \Gamma(z ; p, q)$. In what follows we will simply write $\theta_{1}(u), \Gamma(z), \theta^{(j)}(u)$ and $\theta(z)$ for these functions.

\subsection{Belavin model}

The Belavin model [3] is an integrable lattice model that generalizes Baxter's eight-vertex model $[1,2]$. It is a vertex model, meaning that its spin variables reside on the edges and interact at the vertices of the lattice. These spins take values in $V$, and the local Boltzmann weight for a configuration of four spins placed around a vertex is determined by an operator $R^{\mathrm{B}}: \mathbb{C} \rightarrow \operatorname{End}(V \otimes V)$, the R-matrix of the model.

Belavin's R-matrix $R^{\mathrm{B}}(u)$ is a unique $\operatorname{End}(V \otimes V)$-valued meromorphic function that has simple poles at $u=-\gamma+\mathbb{Z}+\tau \mathbb{Z}$, satisfies the initial condition

$$
R^{\mathrm{B}}(0)=P: v \otimes w \mapsto w \otimes v,
$$

and possesses the $\mathbb{Z}_{N}$-symmetry

$$
R^{\mathrm{B}}(u)=(g \otimes g) R^{\mathrm{B}}(u)(g \otimes g)^{-1}=(h \otimes h) R^{\mathrm{B}}(u)(h \otimes h)^{-1}
$$

and the quasi-periodicity

$$
\begin{aligned}
& R^{\mathrm{B}}(u+1)=\left(g \otimes \mathrm{id}_{V}\right)^{-1} R^{\mathrm{B}}(u)\left(g \otimes \mathrm{id}_{V}\right), \\
& R^{\mathrm{B}}(u+\tau)=q^{1-1 / N}\left(h \otimes \mathrm{id}_{V}\right) R^{\mathrm{B}}(u)\left(h \otimes \mathrm{id}_{V}\right)^{-1} .
\end{aligned}
$$

Here $g, h \in \operatorname{End}(V)$ are matrices such that $g e_{k}=e^{2 \pi \mathrm{i} k / N} e_{k}$ and $h e_{k}=e_{k+1}$, with $e_{N+1}=$ $e_{1}$. The variable $u$ is called the spectral parameter.

In terms of the matrix elements $R^{\mathrm{B}}(u)_{i j}^{k l}$ defined by $R^{\mathrm{B}}(u)\left(e_{i} \otimes e_{j}\right)=\sum_{k, l} R^{\mathrm{B}}(u)_{i j}^{k l} e_{k} \otimes e_{l}$, the R-matrix is given by [35]

$$
R^{\mathrm{B}}(u)_{i j}^{k l}=\delta_{i+j, k+l} \frac{\theta_{1}(\gamma)}{\theta_{1}(u+\gamma)} \frac{\theta^{(k-l)}(u+\gamma)}{\theta^{(k-i)}(\gamma) \theta^{(i-l)}(u)} \frac{\prod_{m=0}^{N-1} \theta^{(m)}(u)}{\prod_{n=1}^{N-1} \theta^{(n)}(0)},
$$


where the indices $i, j, k, l$ are treated modulo $N$. It can be shown that $R^{\mathrm{B}}(u)$ satisfies the unitarity relation

$$
R^{\mathrm{B}}(u) R_{21}^{\mathrm{B}}(-u)=\mathrm{id}_{V \otimes V}
$$

and solves the Yang-Baxter equation

$$
R_{12}^{\mathrm{B}}\left(u_{1}-u_{2}\right) R_{13}^{\mathrm{B}}\left(u_{1}-u_{3}\right) R_{23}^{\mathrm{B}}\left(u_{2}-u_{3}\right)=R_{23}^{\mathrm{B}}\left(u_{2}-u_{3}\right) R_{13}^{\mathrm{B}}\left(u_{1}-u_{3}\right) R_{12}^{\mathrm{B}}\left(u_{1}-u_{2}\right) .
$$

In these equations the subscripts on the R-matrices indicate the space on which they act, e.g., $R_{21}^{\mathrm{B}}(u)=P R^{\mathrm{B}}(u) P$ and $R_{23}^{\mathrm{B}}(u)=\mathrm{id}_{V} \otimes R^{\mathrm{B}}(u)$.

We represent the R-matrix graphically by the crossing of two dashed lines in a dark shaded background:

$$
R^{\mathrm{B}}\left(u_{1}-u_{2}\right)=u_{1}
$$

Each dashed line carries a spectral parameter, and to each segment of a dashed line is assigned a copy of $V$. The R-matrix depends on the difference of the spectral parameters $u_{1}$, $u_{2}$ of the two lines. We may think of the right-hand side as depicting the trajectories of two particles with rapidities $u_{1}$ and $u_{2}$, scattering in $1+1$ spacetime dimensions. The state space of each particle is $V$. The matrix element $R^{\mathrm{B}}\left(u_{1}-u_{2}\right)_{i j}^{k l}$ is the S-matrix element for the scattering process with initial state $\left|u_{1}, i\right\rangle \otimes\left|u_{2}, j\right\rangle$ and final state $\left|u_{1}, k\right\rangle \otimes\left|u_{2}, l\right\rangle$.

In this graphical notation, the unitarity relation (2.9) is expressed as

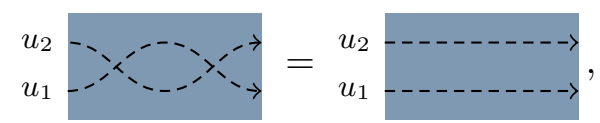

whereas the Yang-Baxter equation (2.10) takes the form of an equality between two configurations of three dashed lines:

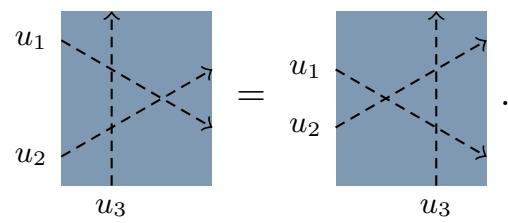

The latter relation states that a three-particle scattering process factorizes into two-particle scattering processes, and the order of this factorization is immaterial.

Now, consider a lattice constructed from dashed lines in a dark shaded background, such as the one shown in figure 3(a). The partition function of the Belavin model on this lattice is defined as follows. For each configuration of states on the edges, we define the corresponding Boltzmann weight to be the product of the R-matrix elements that arise from the crossings. Then, the partition function is the sum of the Boltzmann weights for all possible configurations of states. If the lattice is placed on a surface with boundary, then the states on the edges that intersect the boundary are fixed and not summed over.

Note that if we reverse the directions of the lines in the Yang-Baxter equation (2.13), we end up with the same set of pictures. This means that the transpose $\widetilde{R}^{\mathrm{B}}(u)=R^{\mathrm{B}}(u)^{T}$ 
of $R^{\mathrm{B}}(u)$ is also a solution of the Yang-Baxter equation. We represent it by the crossing of two dashed lines in a light shaded background:

$$
\widetilde{R}^{\mathrm{B}}\left(u_{1}-u_{2}\right)=u_{1} \begin{gathered}
\hat{i} \\
\hdashline \\
u_{2}
\end{gathered}=u_{1}
$$

When $N=2$, Belavin's R-matrix reduces to Baxter's R-matrix for the eight-vertex model. In this case we have $\widetilde{R}^{\mathrm{B}}(u)=R^{\mathrm{B}}(u)$, hence there is no distinction between the two kinds of shading.

\section{$2.2 \quad$ Felder's dynamical R-matrix}

Next, we turn to Felder's dynamical R-matrix for the elliptic quantum group $E_{\tau, \gamma / 2}\left(\mathfrak{s l}_{N}\right)$. This R-matrix first appeared in [10] as the Boltzmann weight of a generalization of the eight-vertex solid-on-solid model [9]. Later, Felder [4, 5] reformulated it as described here.

Compared to Belavin's, Felder's R-matrix $R^{\mathrm{F}}(u, \lambda) \in \operatorname{End}(V \otimes V)$ depends on an additional parameter $\lambda \in \mathfrak{h}^{*}$, called the dynamical variable. The R-matrix satisfies the unitarity relation

$$
R^{\mathrm{F}}(u, \lambda) R_{21}^{\mathrm{F}}(-u, \lambda)=\mathrm{id}_{V \otimes V}
$$

and the dynamical Yang-Baxter equation

$$
\begin{aligned}
& R_{12}^{\mathrm{F}}\left(u_{1}-u_{2}, \lambda-\gamma h_{3}\right) R_{13}^{\mathrm{F}}\left(u_{1}-u_{3}, \lambda\right) R_{23}^{\mathrm{F}}\left(u_{2}-u_{3}, \lambda-\gamma h_{1}\right) \\
& \quad=R_{23}^{\mathrm{F}}\left(u_{2}-u_{3}, \lambda\right) R_{13}^{\mathrm{F}}\left(u_{1}-u_{3}, \lambda-\gamma h_{2}\right) R_{12}^{\mathrm{F}}\left(u_{1}-u_{2}, \lambda\right) .
\end{aligned}
$$

Here $h$ stands for the weight of the relevant state; for example, $R_{12}^{\mathrm{F}}\left(u_{1}-u_{2}, \lambda-\gamma h_{3}\right)$ acts on $v_{1} \otimes v_{2} \otimes v_{3}$ as $R^{\mathrm{F}}\left(u_{1}-u_{2}, \lambda-\gamma \mu_{3}\right) \otimes \mathrm{id} V$ if the weight of $v_{3}$ is $\mu_{3}$. The nonzero matrix elements of $R^{\mathrm{F}}(u, \lambda)$ are given by [36]

$$
R^{\mathrm{F}}(u, \lambda)_{i i}^{i i}=1, \quad R^{\mathrm{F}}(u, \lambda)_{i j}^{i j}=\frac{\theta_{1}(u) \theta_{1}\left(\lambda_{i j}+\gamma\right)}{\theta_{1}(u+\gamma) \theta_{1}\left(\lambda_{i j}\right)}, \quad R^{\mathrm{F}}(u, \lambda)_{i j}^{j i}=\frac{\theta_{1}(\gamma) \theta_{1}\left(u+\lambda_{i j}\right)}{\theta_{1}(u+\gamma) \theta_{1}\left(\lambda_{i j}\right)}
$$

where $i \neq j$ and $\lambda_{i j}=\lambda_{i}-\lambda_{j}$.

Graphically, we represent Felder's R-matrix by the crossing of two dashed lines in an unshaded background:

$$
R^{\mathrm{F}}\left(u_{1}-u_{2}, \lambda\right)=u_{1} \lambda \begin{gathered}
\lambda \\
\vdots \\
\vdots \\
\vdots \\
u_{2}
\end{gathered}
$$

We have marked the upper-left region with $\lambda$ to indicate the dependence on the dynamical variable. As before, the unitarity relation is the equality

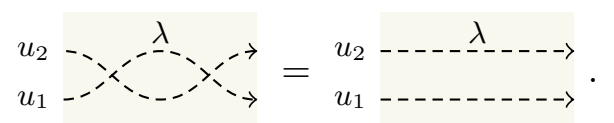


To express the dynamical Yang-Baxter equation (2.16) graphically, we demand that across a dashed line segment, the value of the dynamical variable changes by $\gamma$ times the weight of the state supported on that segment:

$$
\frac{\lambda}{\lambda-\gamma h}
$$

Then we have

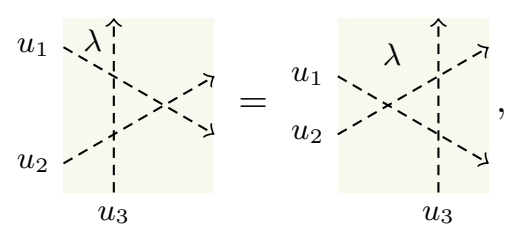

just as in the nondynamical case.

Felder's R-matrix has the property $R^{\mathrm{F}}(u, \lambda)=R^{\mathrm{F}}\left(u, \lambda+\gamma\left(h_{1}+h_{2}\right)\right)$, or

$$
\begin{array}{cc}
\lambda \hat{i} \\
u_{1} \\
\hdashline \vdots & 1 \\
\vdots & 1 \\
u_{2} & \vdots \\
\vdots & u_{2}
\end{array}
$$

Also, the dynamical Yang-Baxter equation holds after the directions of the lines are reversed:

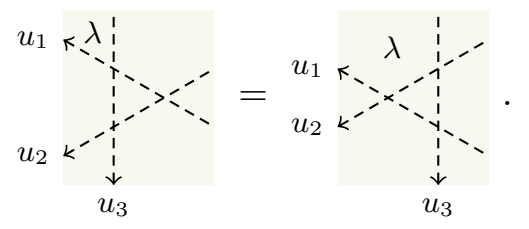

From these relations it follows that the R-matrix ${ }^{\Theta} R^{\mathrm{F}}(u, \lambda)=R^{\mathrm{F}}(u,-\lambda)^{T}$ also satisfies the dynamical Yang-Baxter equation. Note the replacement $\lambda \rightarrow-\lambda$ in the argument. This is necessary to correct the way in which the dynamical variable changes since the lines have been flipped.

In fact, this $\mathrm{R}$-matrix is related to $R^{\mathrm{F}}(u, \lambda)$ by conjugation:

$$
R^{\mathrm{F}}(u,-\lambda)^{T}=\Theta_{1}\left(\lambda-\gamma h_{2}\right) \Theta_{2}(\lambda) R^{\mathrm{F}}(u, \lambda) \Theta_{1}(\lambda)^{-1} \Theta_{2}\left(\lambda-\gamma h_{1}\right)^{-1},
$$

where the matrix $\Theta(\lambda) \in \operatorname{End}(V)$ is given by

$$
\Theta(\lambda)_{j}^{i}=\delta_{j}^{i} \prod_{k(\neq j)} \frac{1}{\theta_{1}\left(\lambda_{k j}\right)} .
$$

In general, if an R-matrix $R(u, \lambda)$ solves the dynamical Yang-Baxter equation, then so does ${ }^{M} R(u, \lambda)=M_{1}\left(\lambda-\gamma h_{2}\right) M_{2}(\lambda) R(u, \lambda) M_{1}(\lambda)^{-1} M_{2}\left(\lambda-\gamma h_{1}\right)^{-1}$ for any invertible matrixvalued function $M(\lambda)$. 


\subsection{Vertex-face correspondence and Jimbo-Miwa-Okado model}

Felder's R-matrix can be obtained from Belavin's by conjugation. Let us define a matrix $\Phi(u, \lambda) \in \operatorname{End}(V)$ by

$$
\Phi(u, \lambda)_{i}^{j}=\theta^{(j)}\left(u-N \lambda_{i}+\frac{N-1}{2}\right) .
$$

Then the two R-matrices are related as follows $[9,10]$ :

$$
R^{\mathrm{B}}\left(u_{1}-u_{2}\right) \Phi_{1}\left(u_{1}, \lambda\right) \Phi_{2}\left(u_{2}, \lambda+\gamma h_{1}\right)=\Phi_{2}\left(u_{2}, \lambda\right) \Phi_{1}\left(u_{1}, \lambda+\gamma h_{2}\right) R^{\mathrm{F}}\left(u_{1}-u_{2}, \lambda\right)^{T} .
$$

We can rewrite this relation as

$$
R^{\mathrm{B}}\left(u_{1}-u_{2}\right) \Psi_{1}\left(u_{1}, \lambda\right) \Psi_{2}\left(u_{2}, \lambda-\gamma h_{1}\right)=\Psi_{2}\left(u_{2}, \lambda\right) \Psi_{1}\left(u_{1}, \lambda-\gamma h_{2}\right) R^{\mathrm{F}}\left(u_{1}-u_{2}, \lambda\right),
$$

where we have introduced

$$
\Psi(u, \lambda)=\Phi(u,-\lambda) \Theta(\lambda)
$$

and used (2.24) to remove the transpose on $R^{\mathrm{F}}$.

The above relation is called the vertex-face correspondence (or vertex-IRF transformation) since it transforms a vertex model to an interaction-round-a-face (IRF) model and vice versa. An IRF model has spins placed on the faces, and assigns a local Boltzmann weight to a configuration of four spins surrounding a vertex. From the point of view of the dual lattice, interaction takes place among spins located round a face.

The model defined by Felder's R-matrix is naturally a vertex model, but it can also be formulated as an IRF model. Once the states are specified on all edges, the values of the dynamical variables living on the faces are determined by rule (2.20) up to overall shifts. Conversely, a consistent assignment of dynamical variables to the faces determines the states on the edges completely. Hence, instead of these states we may think of the dynamical variables as the spin variables of the model. The IRF model thus obtained is the unrestricted $A_{N-1}^{(1)}$ model of Jimbo, Miwa and Okado [10, 26, 27].

\subsection{Bazhanov-Sergeev model}

The last integrable lattice model that we review is the Bazhanov-Sergeev model. This model was introduced in [6] for $N=2$ and subsequently extended to $N \geq 2$ in [7]. It has continuous spin variables taking values in a maximal torus of $\mathrm{SU}(N)$. Accordingly, its Boltzmann weight is given by an infinite-dimensional R-matrix, which is really an integral operator.

For the purpose of describing the Bazhanov-Sergeev model, it is more convenient to switch to multiplicative notation, i.e., from $\tau, \gamma, \lambda_{i}$ to $p, q, z_{i}$ as defined at the beginning of this section. For example, we will write the dynamical Yang-Baxter equation (2.16) as

$$
\begin{aligned}
R_{12}^{\mathrm{F}}\left(\frac{c_{1}}{c_{2}} ; q^{-h_{3}} z\right) R_{13}^{\mathrm{F}}\left(\frac{c_{1}}{c_{3}} ; z\right) R_{23}^{\mathrm{F}}\left(\frac{c_{2}}{c_{3}} ; q^{-h_{1}} z\right) \\
=R_{23}^{\mathrm{F}}\left(\frac{c_{2}}{c_{3}} ; z\right) R_{13}^{\mathrm{F}}\left(\frac{c_{1}}{c_{3}} ; q^{-h_{2}} z\right) R_{12}^{\mathrm{F}}\left(\frac{c_{1}}{c_{2}} ; z\right),
\end{aligned}
$$


where $c_{1}, c_{2}, c_{3}$ are multiplicative spectral parameters and $q^{-h} z$ is the same quantity as $\lambda-\gamma h$ in additive notation.

In our discussions on Belavin's and Felder's R-matrices, we introduced three kinds of backgrounds. Let $n \in \mathbb{Z}$ be a charge that equals 1,0 and -1 in dark shaded, unshaded and light shaded backgrounds, respectively. We define two types of domain walls that separate regions with different values of $n$. Domain walls of one type, which we draw with solid lines, have the property that $n$ increases by 1 as they are crossed from the left to the right when oriented upward. Those of the other type, drawn with dotted lines, change $n$ by -1 instead. We only consider such configurations of domain walls that $n$ stays in the range $|n| \leq 1 .^{2}$

Crossings of domain walls fall into four groups, distinguished by the way in which the surrounding faces are shaded. We assign a spectral parameter to each domain wall, a dynamical variable to each unshaded region, and the following Boltzmann weights to the crossings:

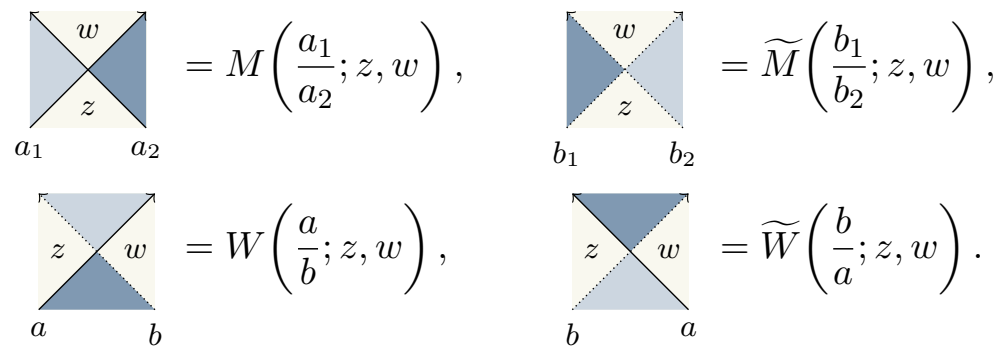

The functions used here are defined by

$$
\begin{array}{rlrl}
M(a ; z, w) & =\frac{\mathcal{I}_{\mathrm{B}}(1 / a ; z, w)}{\Gamma\left(a^{-N}\right)}, & \widetilde{M}(a ; z, w) & =M(a ; w, z), \\
W(a ; z, w) & =\mathcal{I}_{\mathrm{B}}(\sqrt{p q} a ; z, w), & \widetilde{W}(a ; z, w)=W(a ; w, z),
\end{array}
$$

with

$$
\mathcal{I}_{\mathrm{B}}(a ; z, w)=\prod_{i, j} \Gamma\left(a \frac{w_{i}}{z_{j}}\right) .
$$

Note that these are symmetric meromorphic functions with respect to each set of variables $\left(z_{1}, \ldots, z_{N}\right)$ or $\left(w_{1}, \ldots, w_{N}\right)$, and therefore belong to $\mathbb{V} \otimes \mathbb{V}$.

Given a configuration of domain walls on a surface, ${ }^{3}$ we define the associated partition function by the rule that the dynamical variables assigned to the unshaded faces bounded by solid and dotted lines are integrated over. For each such variable $z$, the integration is performed with measure

$$
\int_{\mathbb{T}^{N-1}} \prod_{j=1}^{N-1} \frac{\mathrm{d} z_{j}}{2 \pi \mathrm{i} z_{j}} \mathcal{I}_{\mathrm{V}}(z),
$$

\footnotetext{
${ }^{2}$ The resulting tricolored surfaces are often represented by bipartite graphs whose nodes are placed on shaded regions and connected by edges that go through intersections of domain walls.

${ }^{3}$ In addition to the constraint $|n| \leq 1$, we require that for every bounded face, the domain walls bounding it all go upward locally for some choice of the vertical direction. See [15] for a discussion on this point.
} 
where $\mathbb{T}$ is the unit circle with positive orientation in $\mathbb{C}$ and

$$
\mathcal{I}_{\mathrm{V}}(z)=\frac{(p ; p)_{\infty}^{N-1}(q ; q)_{\infty}^{N-1}}{N !} \prod_{i \neq j} \frac{1}{\Gamma\left(z_{i} / z_{j}\right)}
$$

We do not integrate over the dynamical variables on those faces that contain part of the boundary of the surface. Thus, the partition function is a meromorphic function of these variables.

The crossings of domain walls act on the lattice by concatenation, and their action defines operators on the partition function. The first two crossings in (2.31) represent integral operators $M\left(a_{1} / a_{2}\right), \widetilde{M}\left(b_{1} / b_{2}\right) \in \operatorname{End}(\mathbb{V})$ that act on $f \in \mathbb{V}$ as

$$
\begin{aligned}
& (M(a) f)(w)=\int_{\mathbb{T}^{N-1}} \prod_{j=1}^{N-1} \frac{\mathrm{d} z_{j}}{2 \pi \mathrm{i} z_{j}} \mathcal{I}_{\mathrm{V}}(z) M(a ; z, w) f(z), \\
& (\widetilde{M}(a) f)(w)=\int_{\mathbb{T}^{N-1}} \prod_{k=1}^{N-1} \frac{\mathrm{d} z_{j}}{2 \pi \mathrm{i} z_{j}} \mathcal{I}_{\mathrm{V}}(z) \widetilde{M}(a ; z, w) f(z) .
\end{aligned}
$$

The remaining two crossings represent operators $W(a / b), \widetilde{W}(b / a) \in \operatorname{End}(\mathbb{V} \otimes \mathbb{V})$ that act by multiplication:

$$
\begin{aligned}
& (W(a) f)(z, w)=W(a ; z, w) f(z, w), \\
& (\widetilde{W}(a) f)(z, w)=\widetilde{W}(a ; z, w) f(z, w) .
\end{aligned}
$$

There is no integration here since concatenating these crossings do not produce any unshaded bounded regions. The operator $M$ was introduced in [37] for $N=2$ and [38] for $N \geq 2$. Its action is known as the elliptic Fourier transform.

The unitarity relations hold for domain walls. For example,

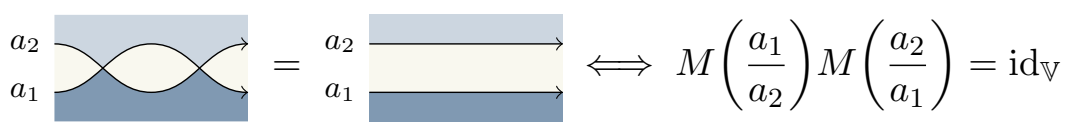

is the inversion relation [38]

$$
\int_{\mathbb{T}^{N-1}} \prod_{j=1}^{N-1} \frac{\mathrm{d} x_{j}}{2 \pi \mathrm{i} x_{j}} \mathcal{I}_{\mathrm{V}}(x) M\left(\frac{a_{2}}{a_{1}} ; z, x\right) M\left(\frac{a_{1}}{a_{2}} ; x, w\right)=\delta(z, w),
$$

where $\delta(z, w)$ is the delta function that equates the two sets of dynamical variables $z$ and $w$ upon integration with respect to measure (2.34). Another unitarity relation

$$
{ }_{b}^{a} \circlearrowright=\begin{aligned}
& a \\
& b
\end{aligned} \Longleftrightarrow \widetilde{W}\left(\frac{b}{a}\right) W\left(\frac{a}{b}\right)=\mathrm{id} \mathbb{V} \otimes \mathbb{V}
$$

is a consequence of the identity $\Gamma(z) \Gamma(p q / z)=1$.

One may hope that in the same vein, the Yang-Baxter equations for domain walls can be established by means of some identities obeyed by the elliptic gamma function. This 
is not the case, unfortunately. The reason is that regions with $|n|>1$ appear in these equations, and the Boltzmann weights appropriate for crossings involving such regions are not known (except in the case $N=2[6,11]$ ).

The Bazhanov-Sergeev model avoids this difficulty by doubling the number of lines. Let us take a pair of solid and dotted lines, and think of it as a single line. We represent it by a double line:

$$
(a, b) \Longrightarrow=\quad b \longrightarrow a
$$

The BSDS R-operator $R^{\mathrm{BSDS}}\left(\left(a_{1}, b_{1}\right),\left(a_{2}, b_{2}\right)\right) \in \operatorname{End}(\mathbb{V} \otimes \mathbb{V})$ is the crossing of two double lines in a dark shaded background:

$$
R^{\mathrm{BSDS}}\left(\left(a_{1}, b_{1}\right),\left(a_{2}, b_{2}\right)\right)=\left(a_{1}, b_{1}\right) \underset{\left(a_{2}, b_{2}\right)}{\stackrel{\prod}{\longrightarrow}}=b_{a_{1}}^{b_{b_{2}} a_{2}} \underset{\mid}{\longrightarrow} .
$$

In terms of the operators defined above, we have

$$
R^{\mathrm{BSDS}}\left(\left(a_{1}, b_{1}\right),\left(a_{2}, b_{2}\right)\right)=\mathbb{P} \widetilde{W}\left(\frac{b_{1}}{a_{2}}\right) M_{2}\left(\frac{a_{1}}{a_{2}}\right) \widetilde{M}_{1}\left(\frac{b_{1}}{b_{2}}\right) W\left(\frac{a_{1}}{b_{2}}\right)
$$

where $\mathbb{P}$ swaps the two sets of variables, $(\mathbb{P} f)(z, w)=f(w, z)$.

The Bazhanov-Sergeev model is therefore a vertex model whose spins are dynamical variables, and placed on a tricolor checkerboard lattice such as the one shown in figure 1(a). Its Yang-Baxter equation

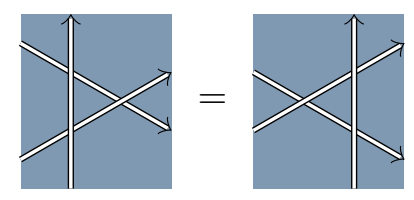

does not involve any undesirable regions, and can be derived from an integral identity for the elliptic gamma function [39-41].

Of course, using a pair of solid and dotted lines we can make another line, which we represent by a thick solid line:

$$
(a, b) \longrightarrow=\begin{aligned}
& a \longrightarrow \\
& b \longrightarrow \ldots \ldots
\end{aligned}
$$

The R-operator defined by the crossing of two thick lines in a light shaded background,

$$
\widetilde{R}^{\mathrm{BS}}\left(\left(a_{1}, b_{1}\right),\left(a_{2}, b_{2}\right)\right)=\left(a_{1}, b_{1}\right) \underset{\left(a_{2}, b_{2}\right)}{\longrightarrow}=a_{b_{1}} \underset{a_{2} b_{2}}{\stackrel{\uparrow}{\longrightarrow}},
$$

is an equally good solution of the Yang-Baxter equation. 


\section{Unification}

In the previous section we introduced dashed lines in shaded and unshaded backgrounds to describe the Belavin and Jimbo-Miwa-Okado models, and solid and dotted lines representing domain walls to formulate the Bazhanov-Sergeev model. In fact, the three kinds of lines can coexist in a single model without spoiling integrability. In this section we construct this integrable lattice model that unifies the three models.

\subsection{Intertwining operators}

The unified model, if exists, should allow dashed lines to cross solid and dotted lines. Conversely, in order to establish the existence of the unified model, we just need to determine what should be assigned to such crossings and show that they are compatible with integrability.

Consider crossings that involve dark shaded and unshaded regions. They define two matrix-valued functions $S(a ; z), S^{\prime}(a ; z) \in \operatorname{End}(V) \otimes \mathbb{V}$ :

$$
\underset{a}{c \uparrow}=S\left(\frac{c}{a} ; z\right), \quad c \underset{z}{c \uparrow}=S^{\prime}\left(\frac{c}{b} ; z\right) \text {. }
$$

Similarly, those involving light shaded and unshaded regions define matrix-valued functions $\widetilde{S}(a ; z), \widetilde{S}^{\prime}(a ; z) \in \operatorname{End}(V) \otimes \mathbb{V}:$

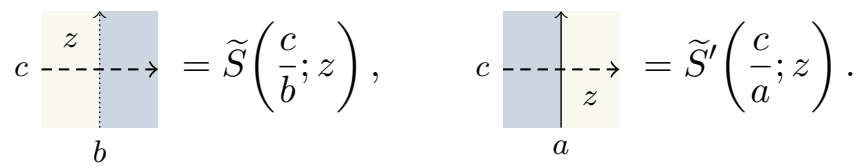

We assume that these functions depend on the spectral parameters of the relevant lines only through their ratio. This is a reasonable assumption since $R^{\mathrm{B}}, R^{\mathrm{F}}$ and $R^{\mathrm{BSDS}}$ all have this property.

A dashed line segment supporting a state $e_{i} \in V$ changes the dynamical variable $\lambda$ by $-\gamma \omega_{i}$ according to the rule shown in (2.20). As such, it acts on the partition function as the inverse of the difference operator

$$
T_{i}: \lambda \mapsto \lambda+\gamma \omega_{i}
$$

The above crossings thus represent the matrices of difference operators $S(c / a), S^{\prime}(c / b)$, $\widetilde{S}(c / b), \widetilde{S}^{\prime}(c / a) \in \operatorname{End}(V \otimes \mathbb{V})$ whose elements are given by

$$
\begin{array}{ll}
S(a)_{i}^{j}=S(a ; z)_{i}^{j} T_{i}^{-1}, & S^{\prime}(a)_{i}^{j}=T_{j}^{-1} S^{\prime}(a ; z)_{i}^{j}, \\
\widetilde{S}(a)_{i}^{j}=\widetilde{S}(a ; z)_{i}^{j} T_{i}^{-1}, & \widetilde{S}^{\prime}(a)_{i}^{j}=T_{j}^{-1} \widetilde{S}^{\prime}(a ; z)_{i}^{j} .
\end{array}
$$

As we will see, $S$ and $S^{\prime}$ intertwine $R^{\mathrm{B}}$ and $R^{\mathrm{F}}$, whereas $\widetilde{S}$ and $\widetilde{S}^{\prime}$ intertwine $\widetilde{R}^{\mathrm{B}}$ and $R^{\mathrm{F}}$. The unified model should be constructed from these intertwining operators, together with the R-matrices (2.11), (2.14) and (2.18) as well as the operators (2.31). 


\subsection{Yang-Baxter equations with one dashed line}

Let us determine the intertwining operators. To this end, we analyze Yang-Baxter equations that involve a single dashed line. Part of this analysis was essentially done in [23, 24]. A similar analysis was carried out for $N=2$ in [8].

First, we look at the following Yang-Baxter equation that contains $S$ and $\widetilde{S}^{\prime}: 4$

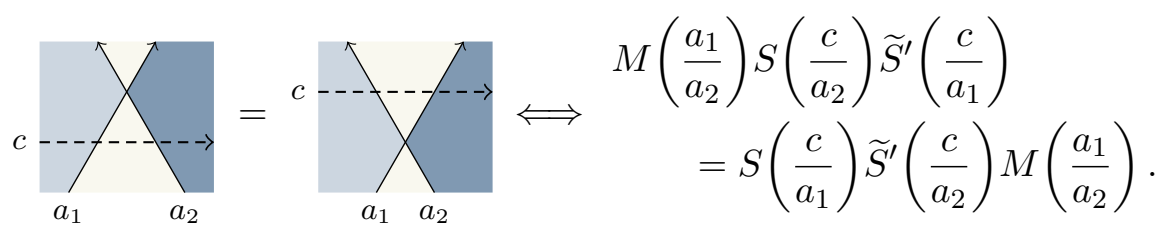

This is a relation between integral operators on $V \otimes \mathbb{V}$. In components, it reads

$$
\begin{aligned}
& \int_{\mathbb{T}^{N-1}} \prod_{l=1}^{N-1} \frac{\mathrm{d} x_{l}}{2 \pi \mathrm{i} x_{l}} \mathcal{I}_{\mathrm{V}}(x) \mathcal{I}_{\mathrm{B}}\left(\frac{a_{2}}{a_{1}} ; x, w\right) \sum_{i, k} S\left(\frac{c}{a_{2}} ; x\right)_{k}^{j} \widetilde{S}^{\prime}\left(\frac{c}{a_{1}} ; T_{k}^{-1} x\right)_{i}^{k} f^{i}\left(T_{k}^{-1} x\right) \\
& =\sum_{i, k} S\left(\frac{c}{a_{1}} ; w\right)_{k}^{j} \widetilde{S}^{\prime}\left(\frac{c}{a_{2}} ; T_{k}^{-1} w\right)_{i}^{k} \int_{\mathbb{T}^{N-1}} \prod_{l=1}^{N-1} \frac{\mathrm{d} z_{l}}{2 \pi \mathrm{i} z_{l}} \mathcal{I}_{\mathrm{V}}(z) \mathcal{I}_{\mathrm{B}}\left(\frac{a_{2}}{a_{1}} ; z, T_{k}^{-1} w\right) f^{i}(z),
\end{aligned}
$$

where $f \in V \otimes \mathbb{V}$ is a $V$-valued function on which the operators act.

For each summand on the left-hand side, let us change the integration variables from $x$ to $z=T_{k}^{-1} x$ so that the same factor $f^{i}(z)$ appears in the integrands of both sides. After this change of variables, the integration is performed with the same measure $\prod_{l=1}^{N-1} \mathrm{~d} z_{l} / 2 \pi \mathrm{i} z_{l}$ as the one used on the right-hand side, but over a different contour given by $\left|T_{k} z_{l}\right|=1$. In order to compare the two sides, we deform this contour to the one given by $\left|z_{l}\right|=1$ or $\left|T_{k} z_{l}\right|=|q|^{\delta_{k l}-1 / N}$. For this deformation to leave the integral unchanged, the integrand should have no simple poles in the domain sandwiched by the two contours, for all $k$ and $l .^{5}$

The poles of the factor $\mathcal{I}_{\mathrm{B}}\left(a_{2} / a_{1} ; T_{k} z, w\right)$ are located at $T_{k} z_{i}=p^{m} q^{n} a_{2} w_{j} / a_{1}$, where $i$, $j=1, \ldots, N$ and $m, n$ are nonnegative integers. None of these poles enters the relevant domain if and only if there exist no pairs $(m, n)$ such that $|q|^{1-1 / N} \leq\left|p^{m} q^{n} a_{2} w_{j} / a_{1}\right| \leq$ $|q|^{-1 / N}$ for some $j$. This is the case if and only if

$$
\left|\frac{a_{2}}{a_{1}} w_{j}\right|<|q|^{1-1 / N}
$$

for all $j$. For the moment we assume that the remaining part of the integrand does not introduce harmful poles either.

Comparing the integrands, we see that for the above equation to hold for any $f \in V \otimes \mathbb{V}$ satisfying this assumption, $S(a ; z)$ and $\widetilde{S}^{\prime}(a ; z)$ should satisfy

$$
\begin{aligned}
& \sum_{k} \mathcal{I}_{\mathrm{V}}\left(T_{k} z\right) \mathcal{I}_{\mathrm{B}}\left(\frac{a_{2}}{a_{1}} ; T_{k} z, w\right) S\left(\frac{c}{a_{2}} ; T_{k} z\right)_{k}^{j} \widetilde{S}^{\prime}\left(\frac{c}{a_{1}} ; z\right)_{i}^{k} \\
&=\sum_{k} S\left(\frac{c}{a_{1}} ; w\right)_{k}^{j} \widetilde{S}^{\prime}\left(\frac{c}{a_{2}} ; T_{k}^{-1} w\right)_{i}^{k} \mathcal{I}_{\mathrm{V}}(z) \mathcal{I}_{\mathrm{B}}\left(\frac{a_{2}}{a_{1}} ; z, T_{k}^{-1} w\right) .
\end{aligned}
$$

\footnotetext{
${ }^{4}$ By $S(a) \widetilde{S}^{\prime}(b)$ we mean an End(V)-valued matrix whose $(i, j)$ component is $\sum_{k} S(a ; z){ }_{k}^{i} T_{k}^{-1} \widetilde{S}^{\prime}(b ; z)_{j}^{k}$.

${ }^{5} \mathrm{In}$ principle, it is possible that the contributions from the simple poles in this domain cancel out. We do not consider this possibility since we want to keep $f$ as general as possible.
} 
It follows that we should have

$$
\begin{aligned}
\mathcal{I}_{\mathrm{V}}\left(T_{i} z\right) \mathcal{I}_{\mathrm{B}}\left(\frac{a_{2}}{a_{1}} ; T_{i} z, T_{j} w\right)\left[S\left(\frac{c}{a_{1}} ; T_{j} w\right)^{-1} S\left(\frac{c}{a_{2}} ; T_{i} z\right)\right]_{i}^{j} \\
=\left[\widetilde{S}^{\prime}\left(\frac{c}{a_{2}} ; w\right) \widetilde{S}^{\prime}\left(\frac{c}{a_{1}} ; z\right)^{-1}\right]_{i}^{j} \mathcal{I}_{\mathrm{V}}(z) \mathcal{I}_{\mathrm{B}}\left(\frac{a_{2}}{a_{1}} ; z, w\right) .
\end{aligned}
$$

Graphically, this equation can be expressed as

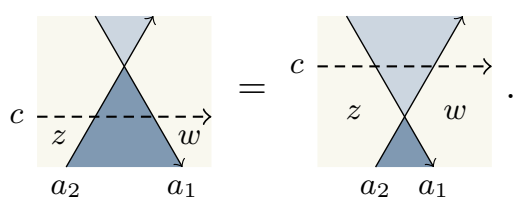

Canceling the elliptic gamma functions that appear on the two sides, we get

$$
\frac{\left[S\left(c / a_{1} ; T_{j} w\right)^{-1} S\left(c / a_{2} ; T_{i} z\right)\right]_{i}^{j}}{\left[\widetilde{S}^{\prime}\left(c / a_{2} ; w\right) \widetilde{S}^{\prime}\left(c / a_{1} ; z\right)^{-1}\right]_{i}^{j}}=\frac{\prod_{l(\neq j)} \theta\left(q^{-1} a_{2} w_{l} / a_{1} z_{i}\right)}{\prod_{k(\neq i)} \theta\left(a_{2} w_{j} / a_{1} z_{k}\right)} \prod_{k(\neq i)} \frac{\theta\left(z_{i} / z_{k}\right)}{\theta\left(q^{-1} z_{k} / z_{i}\right)} .
$$

To solve the above equation, we use the matrices $\Phi(u, \lambda)$ and $\Psi(u, \lambda)$ introduced in section 2.3 in the context of the vertex-face correspondence. We have [32]

$$
\left[\Phi(v, \mu)^{-1} \Phi(u, \lambda)\right]_{i}^{j}=\frac{\theta_{1}\left(v+(u-v) / N+\mu_{j}-\lambda_{i}\right)}{\theta_{1}(v)} \prod_{l(\neq j)} \frac{\theta_{1}\left((u-v) / N+\mu_{l}-\lambda_{i}\right)}{\theta_{1}\left(\mu_{l}-\mu_{j}\right)} .
$$

Thus, if we set

$$
\begin{aligned}
S(a ; z) & =a^{-N / 2} \Psi(u, \lambda), \\
\widetilde{S}^{\prime}(a ; z) & =a^{-N / 2} Z^{N / 2} \Phi(u, \lambda)^{T},
\end{aligned}
$$

with $a=e^{2 \pi \mathrm{i} u / N}$ and $Z=\operatorname{diag}\left(z_{1}, \ldots, z_{N}\right)$, then they satisfy

$$
\begin{gathered}
{\left[S(b ; w)^{-1} S(a ; z)\right]_{i}^{j}=\frac{\theta\left(b^{-N} b w_{j} / a z_{i}\right)}{\theta\left(b^{-N}\right)} \frac{\prod_{l(\neq j)} \theta\left(b w_{l} / a z_{i}\right)}{\prod_{k(\neq i)} \theta\left(z_{k} / z_{i}\right)}} \\
{\left[\widetilde{S}^{\prime}(a ; w) \widetilde{S}^{\prime}(b ; z)^{-1}\right]_{i}^{j}=\frac{\theta\left(b^{-N} b w_{j} / a z_{i}\right)}{\theta\left(b^{-N}\right)} \prod_{k(\neq i)} \frac{\theta\left(b w_{j} / a z_{k}\right)}{\theta\left(z_{i} / z_{k}\right)}}
\end{gathered}
$$

and solve (3.12). We have chosen the normalization factor $a^{-N / 2}$ so as to remove factors of $a / b$ from these formulas.

Let us check the validity of the contour deformation for this solution. Apart from the function $f^{i}(z)$, the potentially dangerous factors in the integrand on the left-hand side of (3.7) are $\mathcal{I}_{\mathrm{V}}\left(T_{k} z\right)$ as well as $1 / \prod_{l(\neq k)} \theta\left(q z_{k} / z_{l}\right)$ contained in $S\left(c / a_{2} ; T_{k} z\right)_{k}^{j}$. Their product consists of factors of the form $\Gamma(x) / \Gamma(x / q)$ or $1 / \Gamma(x) \Gamma(1 / x)$ with $x=z_{i} / z_{j}$ for some $i, j$, but neither of these functions has poles except at $x=0$. Thus, the contour deformation is justified as long as inequality (3.8) is satisfied and $f(z)$ has no simple poles in the domain swept out by the deformation. 
Next, we analyze the Yang-Baxter equation

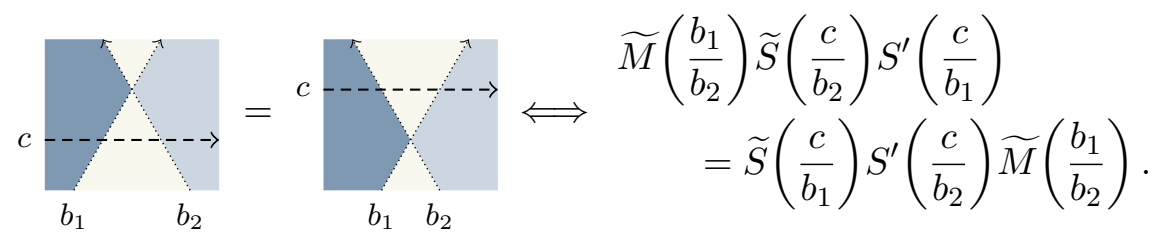

The same contour deformation argument as above leads to the condition

$$
\begin{aligned}
\mathcal{I}_{\mathrm{V}}\left(T_{i} z\right) \mathcal{I}_{\mathrm{B}}\left(\frac{b_{2}}{b_{1}} ; T_{j} w, T_{i} z\right)\left[\widetilde{S}\left(\frac{c}{b_{1}} ; T_{j} w\right)^{-1} \widetilde{S}\left(\frac{c}{b_{2}} ; T_{i} z\right)\right]_{i}^{j} \\
=\left[S^{\prime}\left(\frac{c}{b_{2}} ; w\right) S^{\prime}\left(\frac{c}{b_{1}} ; z\right)^{-1}\right]_{i}^{j} \mathcal{I}_{\mathrm{V}}(z) \mathcal{I}_{\mathrm{B}}\left(\frac{b_{2}}{b_{1}} ; w, z\right),
\end{aligned}
$$

or graphically,

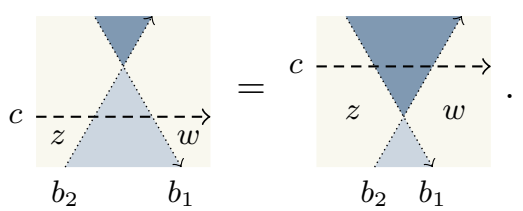

From this equation we get

$$
\begin{aligned}
\frac{\left[\widetilde{S}\left(c / b_{1} ; T_{j} w\right)^{-1} \widetilde{S}\left(c / b_{2} ; T_{i} z\right)\right]_{i}^{j}}{\left[S^{\prime}\left(c / b_{2} ; w\right) S^{\prime}\left(c / b_{1} ; z\right)^{-1}\right]_{i}^{j}} & =\frac{\prod_{k(\neq i)} \theta\left(q^{-1} b_{2} z_{k} / b_{1} w_{j}\right)}{\prod_{l(\neq j)} \theta\left(b_{2} z_{i} / b_{1} w_{l}\right)} \prod_{k(\neq i)} \frac{\theta\left(z_{i} / z_{k}\right)}{\theta\left(q^{-1} z_{k} / z_{i}\right)} \\
& =\left(\frac{z_{i}}{w_{j}}\right)^{N} \frac{\left[\widetilde{S}^{\prime}\left(t c / b_{1} ; T_{j} w\right) \widetilde{S}^{\prime}\left(t c / b_{2} ; T_{i} z\right)^{-1}\right]_{i}^{j}}{\left[S\left(t c / b_{2} ; w\right)^{-1} S\left(t c / b_{1} ; z\right)\right]_{i}^{j}},
\end{aligned}
$$

where $t \in \mathbb{C}$ is an arbitrary parameter.

Finally, the Yang-Baxter equation

$$
\begin{aligned}
& \sum_{a}=\begin{array}{l}
c \\
b
\end{array}=\begin{array}{l}
W\left(\frac{a}{b}\right) S^{\prime}\left(\frac{c}{b}\right) S\left(\frac{c}{a}\right) \\
=\widetilde{S}^{\prime}\left(\frac{c}{a}\right) \widetilde{S}\left(\frac{c}{b}\right) W\left(\frac{a}{b}\right)
\end{array}
\end{aligned}
$$

gives the relation

$$
\begin{aligned}
\mathcal{I}_{\mathrm{B}}\left(\sqrt{p q} \frac{a}{b} ; z, T_{j} w\right)\left[S^{\prime}\left(\frac{c}{b} ; w\right) S\left(\frac{c}{a} ; z\right)\right]_{i}^{j} \\
=\left[\widetilde{S}^{\prime}\left(\frac{c}{a} ; w\right) \widetilde{S}\left(\frac{c}{b} ; z\right)\right]_{i}^{j} \mathcal{I}_{\mathrm{B}}\left(\sqrt{p q} \frac{a}{b} ; T_{i}^{-1} z, w\right) .
\end{aligned}
$$

We can rewrite it as

$$
\begin{aligned}
\frac{\left[\widetilde{S}^{\prime}(c / a ; w) \widetilde{S}(c / b ; z)\right]_{i}^{j}}{\left[S^{\prime}(c / b ; w) S(c / a ; z)\right]_{i}^{j}} & =\frac{\prod_{k(\neq i)} \theta\left(q^{-1 / N} \sqrt{p q} a w_{j} / b z_{k}\right)}{\prod_{l(\neq j)} \theta\left(q^{-1 / N} \sqrt{p q} a w_{l} / b z_{i}\right)} \\
& =(-1)^{N-1} \frac{\left[\widetilde{S}^{\prime}(c / a ; w) \widetilde{S}^{\prime}\left(q^{-1 / N} \sqrt{p q} c / b ; z\right)^{-1} Z^{N}\right]_{i}^{j}}{\left[S\left(q^{-1 / N} \sqrt{p q} c / b ; w\right)^{-1} S(c / a ; z)\right]_{i}^{j}} .
\end{aligned}
$$


The two equations (3.21) and (3.24) are solved by

$$
\begin{aligned}
S^{\prime}(a ; z) & =S\left(q^{-1 / N} \sqrt{p q} a ; z\right)^{-1}, \\
\widetilde{S}(a ; z) & =(-1)^{N-1} \widetilde{S}^{\prime}\left(q^{-1 / N} \sqrt{p q} a ; z\right)^{-1} Z^{N} .
\end{aligned}
$$

The remaining Yang-Baxter equations with one dashed line follow from those considered above.

The contour deformation argument used in the analysis of relation (3.18) is valid for the above solution, provided that

$$
\left|\frac{b_{1}}{b_{2}} w_{j}\right|>|q|^{-1 / N}
$$

for all $j$ and the two sides of the relation act on functions that have no simple poles in the deformation domain. This inequality ensures that $\mathcal{I}_{\mathrm{B}}\left(b_{2} / b_{1} ; w, T_{k} z\right)$ has no simple poles in the domain. To see that the other relevant factors have no simple poles there either, we calculate $\sum_{j} \widetilde{S}^{\prime}(a ; w)_{j}^{l} \mathcal{I}_{\mathrm{V}}\left(T_{k} z\right) \widetilde{S}\left(c / b_{2} ; T_{k} z\right)_{k}^{j}$ and $\sum_{i} S^{\prime}\left(c / b_{1} ; z\right)_{i}^{k} S(a ; w)_{l}^{i}$ using formulas (3.16) and (3.17). Recalling that $\mathcal{I}_{\mathrm{V}}\left(T_{k} z\right) / \prod_{l(\neq k)} \theta\left(q z_{k} / z_{l}\right)$ has no poles in the domain under consideration, we find that these quantities do not have harmful poles in $z$. Hence, the same is true for $\mathcal{I}_{\mathrm{V}}\left(T_{k} z\right) \widetilde{S}\left(c / b_{2} ; T_{k} z\right)_{k}^{j}$ and $S^{\prime}\left(c / b_{1} ; z\right)_{i}^{k}$.

\subsection{Yang-Baxter equations with two dashed lines}

Now we move on to the Yang-Baxter equations that contain two dashed lines. Those with a solid line are

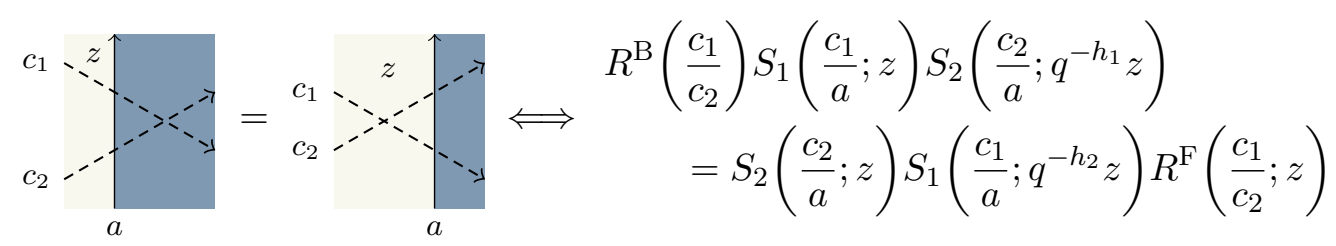

and

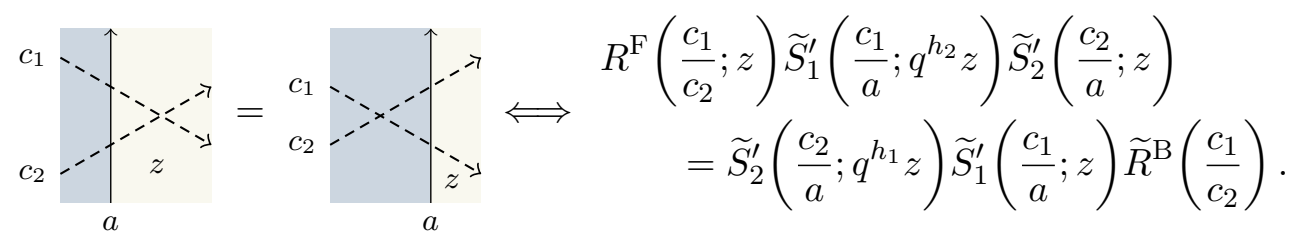

These equations describe the vertex-face correspondence discussed in section 2.3. The first one is relation (2.28), while the second is a consequence of relation (2.27) and the fact that $R^{\mathrm{F}}(a, z)_{i j}^{k l}=0$ unless $\{i, j\}=\{k, l\}$.

We can replace the solid lines above with dotted ones and obtain two more relations. Let us look at the Yang-Baxter equation

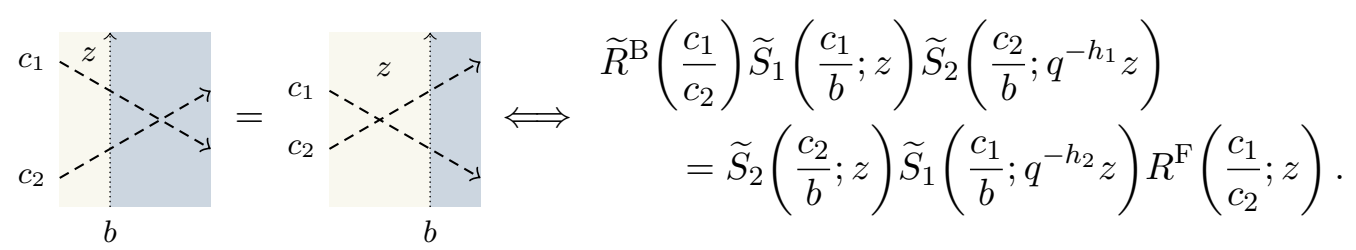


It can be rewritten as

$$
\begin{aligned}
\sum_{m, n} \widetilde{S}^{\prime}\left(q^{-1 / N} \sqrt{p q} \frac{c_{1}}{b} ; T_{l}^{-1} z\right)_{m}^{k} \widetilde{S}^{\prime}\left(q^{-1 / N} \sqrt{p q} \frac{c_{2}}{b} ; z\right)_{n}^{l} \widetilde{R}^{\mathrm{B}}\left(\frac{c_{1}}{c_{2}}\right)_{i j}^{m n} \\
=\sum_{m, n} R^{\mathrm{F}}\left(\frac{c_{1}}{c_{2}} ; z\right)_{m n}^{k l} \widetilde{S}^{\prime}\left(q^{-1 / N} \sqrt{p q} \frac{c_{2}}{b} ; T_{m}^{-1} z\right)_{j}^{n} \widetilde{S}^{\prime}\left(q^{-1 / N} \sqrt{p q} \frac{c_{1}}{b} ; z\right)_{i}^{m} .
\end{aligned}
$$

Replacing $z$ with $T_{k} T_{l} z$ and using the identity

$$
\widetilde{S}^{\prime}\left(a ; T_{j} z\right)_{i}^{j}={\widetilde{S^{\prime}}}^{\prime}\left(q^{-1+1 / N} a ; z\right)_{i}^{j},
$$

we see that this equation reduces to the intertwining relation (3.29).

The last Yang-Baxter equation with two dashed lines,

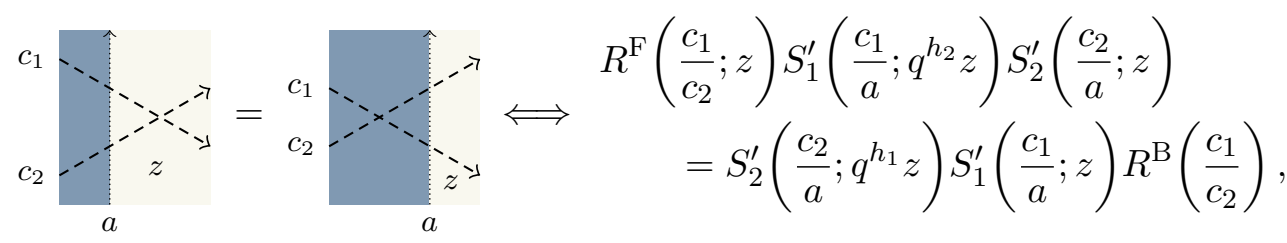

can be verified in a similar manner. An easier way is to make use of the relation

$$
\uparrow_{a_{b}}^{\uparrow} \rightarrow=\frac{\theta\left(\left(b^{\prime} / c\right)^{N}\right)}{\theta\left(q\left(b^{\prime} / c\right)^{N}\right)} \times c-\uparrow-\rightarrow \quad b^{\prime}=\frac{b}{\sqrt{p q}},
$$

which follows from the identity $\left[S\left(a ; T_{j} z\right)^{-1}\right]_{i}^{j}=\left[S\left(q^{-1 / N} a ; z\right)^{-1}\right]_{i}^{j} \theta\left(q a^{-N}\right) / \theta\left(a^{-N}\right)$. This relation and the intertwining relation (3.28) imply

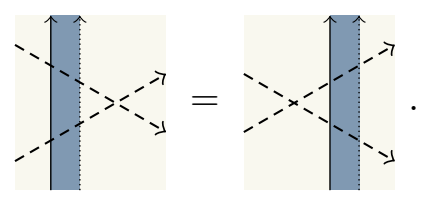

Moving the solid line on the right-hand side past the crossing of the dashed lines, we deduce that relation (3.33) holds.

\section{$3.4 \quad$ L-operators and elliptic quantum groups}

In our framework, an L-operator is constructed from two intertwining operators composed in $V$ in a way consistent with shading. The Yang-Baxter equations with one dashed line studied in section 3.2 may be thought of as describing the action of the operators $M, \widetilde{M}$ and $W$ on L-operators.

An important L-operator $L^{\mathrm{B}}(a, b) \in \operatorname{End}(V \otimes \mathbb{V})$ is given by the crossing of a dashed line and a double line (2.43) in a dark shaded background:

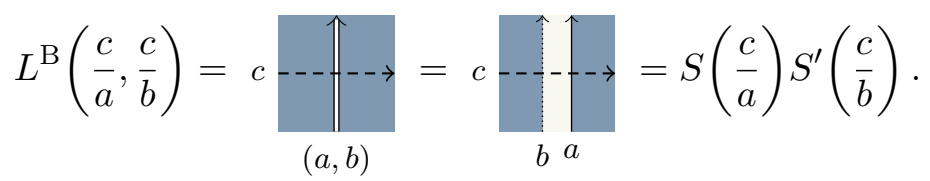


This L-operator was constructed in [23-25]. It satisfies two Yang-Baxter equations,

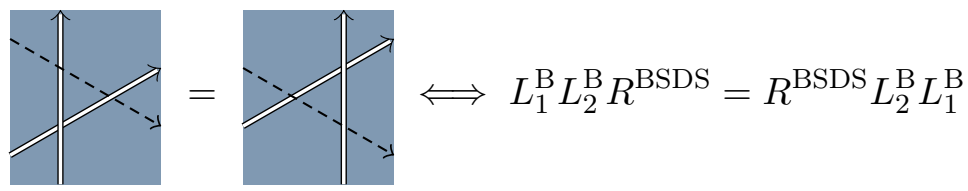

and

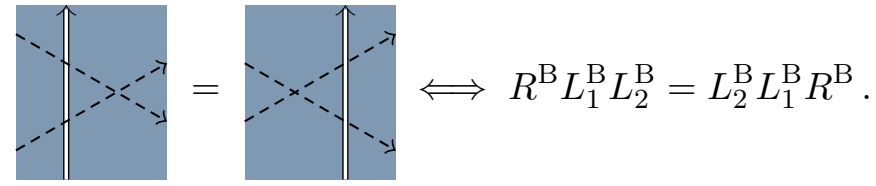

By definition, an L-operator for an R-matrix is an operator that satisfies an RLL relation of this kind together with that R-matrix. We see that $L^{\mathrm{B}}$ is an L-operator for both Belavin's R-matrix and the BSDS R-operator.

In a certain trigonometric limit, the Bazhanov-Sergeev model for $N=2$ reduces to the chiral Potts model [6]. On the other hand, the Belavin model for $N=2$ is the eightvertex model, which becomes the six-vertex model in this limit. Therefore, what we have just found is an elliptic counterpart of the fact that a single L-operator satisfies two RLL relations, one for the chiral Potts model and another one for the six-vertex model [22]. For $N>2$, our result should generalize the relation established in [42].

Placing the crossing of a dashed line and a thick line (2.47) in an unshaded background, we obtain another L-operator $L^{\mathrm{F}}(a, b) \in \operatorname{End}(V \otimes \mathbb{V} \otimes \mathbb{V})$. Let $z$ and $w$ be the dynamical variables for the first and second factors of $\mathbb{V}$, respectively, and denote by $T_{z, i}$ the difference operator (3.3) acting on functions of $z$. Then, $L^{\mathrm{F}}(a, b)$ is a matrix of difference operators with elements

$$
L^{\mathrm{F}}(a, b)_{i}^{j}=T_{w, j}^{-1} L^{\mathrm{F}}(a, b ; z, w)_{i}^{j} T_{z, i}^{-1},
$$

where the matrix-valued function $L^{\mathrm{F}}(a, b ; z, w)$ is given by

$$
L^{\mathrm{F}}\left(\frac{c}{a}, \frac{c}{b} ; z, w\right)=c \underset{(a, b)}{z-\uparrow_{w}^{--\rightarrow}}=c \underset{a_{b}}{-\underset{w}{-} \rightarrow}=S^{\prime}\left(\frac{c}{b} ; w\right) S\left(\frac{c}{a} ; z\right) .
$$

This L-operator satisfies an RLL relation with Felder's R-matrix:

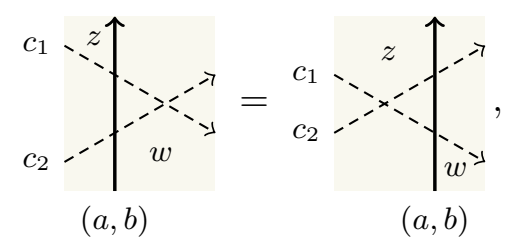

or more explicitly,

$$
\begin{aligned}
R^{\mathrm{F}}\left(\frac{c_{1}}{c_{2}} ; w\right) L_{1}^{\mathrm{F}}\left(\frac{c_{1}}{a}, \frac{c_{1}}{b} ; z, q^{h_{2}} w\right) L_{2}^{\mathrm{F}}\left(\frac{c_{2}}{a}, \frac{c_{2}}{b} ; q^{-h_{1}} z, w\right) \\
=L_{2}^{\mathrm{F}}\left(\frac{c_{2}}{a}, \frac{c_{2}}{b} ; z, q^{h_{1}} w\right) L_{1}^{\mathrm{F}}\left(\frac{c_{1}}{a}, \frac{c_{1}}{b} ; q^{-h_{2}} z, w\right) R^{\mathrm{F}}\left(\frac{c_{1}}{c_{2}} ; z\right) .
\end{aligned}
$$


Note that $L^{\mathrm{F}}$ is obtained from $L^{\mathrm{B}}$ by an interchange of the intertwining operators in the product.

The last relation leads to the notion of the elliptic quantum group $E_{\tau, \gamma / 2}\left(\mathfrak{s l}_{N}\right)[4,5]$. Set $L(u)=L^{\mathrm{F}}(c / a, c / b)$ with $c=e^{2 \pi \mathrm{i} u / N}$, and let $\lambda, \mu$ be additive dynamical variables corresponding to $z, w$, respectively. Then, $L(u)$ act on functions $f(\lambda), g(\mu)$ by

$$
L(u)_{i}^{j} f(\lambda)=f\left(\lambda-\gamma \omega_{i}\right) L(u)_{i}^{j}, \quad L(u)_{i}^{j} g(\mu)=g\left(\mu-\gamma \omega_{j}\right) L(u)_{i}^{j}
$$

and satisfies

$$
\sum_{k, l} R^{\mathrm{F}}\left(u_{1}-u_{2}, \mu\right)_{k l}^{m n} L\left(u_{1}\right)_{i}^{k} L\left(u_{2}\right)_{j}^{l}=\sum_{k, l} R^{\mathrm{F}}\left(u_{1}-u_{2}, \lambda\right)_{i j}^{k l} L\left(u_{2}\right)_{l}^{n} L\left(u_{1}\right)_{k}^{m}
$$

As an algebra, $E_{\tau, \gamma / 2}\left(\mathfrak{s l}_{N}\right)$ is generated by meromorphic functions of $\lambda$ and those of $\mu$, and the matrix elements of an L-operator $L(u)$ and its inverse $L^{-1}(u)$, obeying these relations. It is further endowed with the structure of an $\mathfrak{h}$-Hopf algebroid [43-45].

In fact, $L^{\mathrm{F}}$ gives a representation of $E_{\tau, \gamma / 2}\left(\mathfrak{s l}_{N}\right)$. Let us introduce an $\mathfrak{h}$-module

$$
W=\bigoplus_{\mu \in \mu_{0}+\Lambda} \mathbb{C} w_{\mu}
$$

where $\mu_{0} \in \mathfrak{h}^{*}$ is a parameter, $\Lambda$ is the root lattice of $\mathfrak{s l}_{N}$, and $w_{\mu}$ is a generator of weight $\mu$. If we define $L(u, \lambda) \in \operatorname{End}(V \otimes W)$ by

$$
L(u, \lambda)\left(e_{i} \otimes w_{\mu}\right)=\sum_{j} L^{F}\left(\frac{c}{a}, \frac{c}{b} ; z, q^{-\mu-\omega_{i}} z\right)_{i}^{j} e_{j} \otimes w_{\mu+\omega_{i}-\omega_{j}},
$$

then the RLL relation (3.42) implies

$$
\begin{array}{r}
R_{12}^{\mathrm{F}}\left(u_{1}-u_{2}, \lambda-\gamma h_{3}\right) L_{13}\left(u_{1}, \lambda\right) L_{23}\left(u_{2}, \lambda-\gamma h_{1}\right) \\
=L_{23}\left(u_{2}, \lambda\right) L_{13}\left(u_{1}, \lambda-\gamma h_{2}\right) R_{12}^{\mathrm{F}}\left(u_{1}-u_{2}, \lambda\right) .
\end{array}
$$

A diagonalizable $\mathfrak{h}$-module $W$ and an $\operatorname{End}(V \otimes W)$-valued meromorphic function $L(u, \lambda)$ that commutes with the action of $\mathfrak{h}$ and obeys this relation constitute a representation of $E_{\tau, \gamma / 2}\left(\mathfrak{s l}_{N}\right)[4,5]$.

\section{Surface defects and elliptic quantum groups}

The proposal of this paper is that the integrable lattice model formulated in the previous section can be realized by intersecting branes in string theory. In turn, this proposal implies that a family of surface defects in four-dimensional $\mathcal{N}=1$ supersymmetric field theories correspond to transfer matrices constructed from L-operators for elliptic quantum groups and variants thereof. In this section we discuss the brane construction and this correspondence. We provide evidence for the proposal by computing some classes of transfer matrices and showing that they reproduce known gauge theory results. 

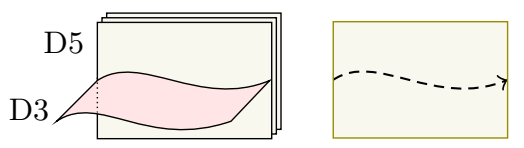

Figure 4. A D3-brane ending on the D5-branes creates a dashed line.

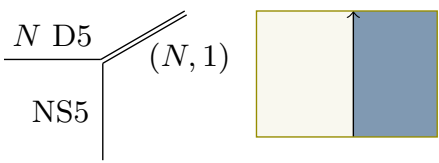

(a)

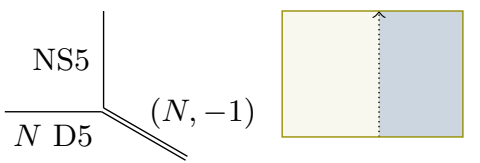

(b)

Figure 5. An NS5-brane combines with $N$ D5-branes to form an $(N, 1)$ or $(N,-1) 5$-brane.

\subsection{Brane construction}

A brane construction of the Bazhanov-Sergeev model was found in [13], and a closely related construction of the eight-vertex model was proposed in [11]; see [15] for a review. The brane construction of the integrable lattice model in question is a natural extension of these constructions.

Consider a stack of $N$ coincident D5-branes supported on $\mathbb{R}^{3,1} \times \Sigma \times 0$ in type IIB string theory in spacetime $\mathbb{R}^{3,1} \times T^{*} \Sigma \times \mathbb{R}^{2}$, where $\Sigma$ is a compact surface embedded in the cotangent bundle $T^{*} \Sigma$ as the zero section. The surface $\Sigma$ wrapped by the D5-branes becomes an unshaded background surface on which a lattice is placed.

To draw a lattice on $\Sigma$, we introduce D3-branes and NS5-branes. Take a D3-brane that ends on the D5-branes along a curve $C$ on $\Sigma$ and is supported on $\mathbb{R}^{1,1} \times \Sigma_{C} \times 0$, where $\Sigma_{C}$ is a suitably chosen surface in $T^{*} \Sigma$ such that $\Sigma_{C} \cap \Sigma=C$. The curve $C$ represents a dashed line (figure 4). Similarly, NS5-branes intersecting the D5-branes create solid and dotted lines. The difference from the D3-brane case is that an NS5-brane cannot simply terminate on the D5-branes. Rather, these 5-branes merge into a bound state, either an $(N, 1) 5$-brane (figure $5(\mathrm{a}))$ or an $(N,-1) 5$-brane (figure $5(\mathrm{~b})$ ), depending on the relative positions of the 5-branes. Therefore, the curve along which the NS5-brane and the D5branes meet is a domain wall on $\Sigma$, which separates a D5-brane region and an $(N, \pm 1)$ 5 -brane region. In our graphical notation, $(N, 1)$ and $(N,-1) 5$-brane regions are indicated by dark and light shading, respectively.

After making a lattice on $\Sigma$ with D3- and NS5-branes, we perform a Wick rotation and compactify $\mathbb{R}^{4}$ to the quotient $\mathcal{M}_{p, q}$ of $\mathbb{C}^{2} \backslash(0,0)$ by the equivalence relation $\left(z_{1}, z_{2}\right) \sim$ $\left(p z_{1}, q z_{2}\right)$. We put the D3-branes on the submanifold $\left\{z_{2}=0\right\}$ of $\mathcal{M}_{p, q}$, which is an elliptic curve $E_{p}$ with modulus $p=e^{2 \pi \mathrm{i} \tau}$. Computing the partition function of the brane system in this new spacetime, we obtain a supersymmetric index [46-49]. It is identified with the partition function of the integrable lattice model defined in the previous section, placed on this lattice.

The supersymmetric index is protected against continuous changes of various parameters of the theory. In the present case, these parameters include the shapes of the D3and NS5-branes. Hence, the partition function of the lattice model is invariant under continuous deformations of lattice lines; it is a topological invariant of the lattice. 
The Yang-Baxter equation states that the partition function remains unchanged as one line moves past the intersection of two other lines. This is a stronger statement than the topological invariance, which does not rule out the possibility of a phase transition occurring when the three lines meet at a point. The absence of such a phase transition follows from string dualities. Topologically, $\mathcal{M}_{p, q}$ is $S^{1} \times S^{3}$. If we apply T-duality along $S^{1}$, we obtain a dual brane system in type IIA string theory, which we can further lift to M-theory. These duality operations turn the D5-branes into M5-branes supported on $S^{3} \times \Sigma \times S^{1}$, where the last $S^{1}$ is the M-theory circle, the 11th dimension that emerged in the process. On the other hand, the D3- and NS5-branes are mapped to M2- and M5-branes, respectively. Since they are generically supported at separate points on the M-theory circle, the seemingly singular situation of three lines meeting at a point is rendered nonsingular in the M-theory picture.

The emergent extra dimension does not only ensure that the Yang-Baxter equation holds, but also provides spectral parameters [19, 20]: the coordinates of the M2- and M5branes on the M-theory circle. Together, the Yang-Baxter equation and the presence of spectral parameters imply the integrability of the lattice model.

In the original frame, the spectral parameters are the holonomies of the $\mathrm{U}(1)$ gauge fields on the NS5-branes and those of the dual U(1) gauge fields on the D3-branes around $S^{1}$ in $\mathcal{M}_{p, q} \simeq S^{1} \times S^{3}$. Likewise, the dynamical variables come from the holonomies of the $\mathrm{SU}(N)$ gauge fields on the D5-brane (i.e. unshaded) regions. The $\mathrm{SU}(N)$ gauge fields are dynamical and integrated over in the path integral, except for those supported in regions that contain part of the boundary of $\Sigma$; on the boundary the 5-branes end on 7-branes, and boundary conditions freeze the dynamics. The partition function is therefore a function of the holonomies of the nondynamical gauge fields. Due to the $\mathrm{SU}(N)$ flavor symmetries, however, it only depends on these holonomies through their conjugacy classes, which are specified by diagonal matrices up to permutations of the entries.

As we see, this brane construction naturally leads to circle-valued spectral parameters, though they can be analytically continued to complex numbers. If we wish to get complex spectral parameters directly, instead of $\mathcal{M}_{p, q}$ we can use $E_{p} \times_{q} \mathbb{C}$, the fibration of $\mathbb{C}$ over $E_{p}$ such that the fiber is rotated by angle $\operatorname{Re} \gamma$ or $\operatorname{Im} \gamma$ as it goes around either cycle of $E_{p}$. The D3-branes are wrapped around $E_{p}$ and supported at the origin of $\mathbb{C}$. To understand the geometric meaning of the spectral parameters in this case, we apply S-duality and then T-duality on each cycle of $E_{p}$. This chain of dualities converts the D3-branes to D1-branes and the NS5-branes to D3-branes, all supported at points on the dual curve $\widetilde{E}_{p}$. The coordinates of these points provide spectral parameters valued in $\widetilde{E}_{p}$. A similar remark applies to dynamical variables.

According to $[50,51]$, the partition function on $E_{p} \times_{q} \mathbb{C}$ (with a Neumann boundary condition imposed on chiral multiplets) takes the identical form as that on $\mathcal{M}_{p, q}$, except that the factor $\sqrt{p q}$ that appears in the elliptic gamma functions are replaced with $\sqrt{q}$ and the dynamical variables are integrated over different contours. Thus, we expect that this change of geometry affects neither the R-matrices assigned to the crossings of dashed lines nor the intertwining operators, apart from simple replacement of $\sqrt{p q}$ with $\sqrt{q}$.

While $E_{p} \times_{q} \mathbb{C}$ may be a more natural choice from the point of view of elliptic lattice models, the use of $\mathcal{M}_{p, q}$ has an advantage. In the above construction we have placed D3- 


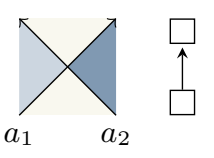

(a)

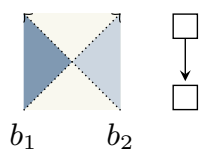

(b)

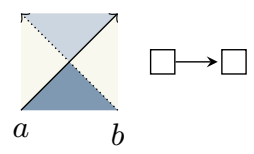

(c)

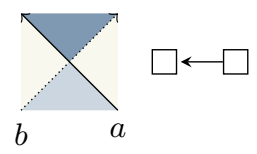

(d)

Figure 6. The rule for assigning a quiver to a tricolor lattice.

branes on the surface $\left\{z_{2}=0\right\}$. However, we may also wrap them on the other distinguished surface, $\left\{z_{1}=0\right\}$. In other words, there are really two types of dashed lines, differing in the support of the D3-brane. Consequently, the Bazhanov-Sergeev model admits two sets of L-operators related by an interchange of $p$ and $q$, leading to an elliptic version [52] of the modular double of quantum groups [53].

\section{2 $\mathcal{N}=1$ supersymmetric quiver gauge theories and surface defects}

In practice, the supersymmetric index of the brane system discussed above is computed in the four-dimensional effective field theory. For a moment, let us decompactify $\mathcal{M}_{p, q}$ to $\mathbb{R}^{4}$ and suppose there are no D3-branes. Since $\Sigma$ is compact, the theory that governs the low-energy dynamics on the 5-branes is a four-dimensional theory formulated on $\mathbb{R}^{4}$.

This theory is an $\mathcal{N}=1$ supersymmetric gauge theory described by a quiver diagram drawn on $\Sigma[17,54]$. In the absence of D3-branes, the brane configuration is encoded in a tricolor lattice on $\Sigma$. To obtain the quiver from the lattice, we place a node on each unshaded face and connect the nodes by arrows according to the rule illustrated in figure 6 . A node is frozen if it is placed on a face that intersects the boundary. Each unfrozen node represents an $\mathrm{SU}(N)$ gauge group, while each frozen one represents an $\mathrm{SU}(N)$ flavor group. Each arrow represents a matter field, more precisely a chiral multiplet transforming in the fundamental representation under the $\mathrm{SU}(N)$ group located at its head and in the antifundamental representation under the $\mathrm{SU}(N)$ group at its tail. The matter fields are also charged under U(1) flavor symmetries associated with NS5-branes.

Now we compactify $\mathbb{R}^{4}$ to $\mathcal{M}_{p, q}$ again and compute the partition function of the gauge theory on $\mathcal{M}_{p, q}$ to obtain the supersymmetric index. Thanks to its protected nature, the index can be evaluated in the weak coupling limit where the path integral reduces to a finite-dimensional integral. The resulting integral [46] is the partition function of the corresponding lattice model on the tricolor lattice, as defined in section 2.4.

Let us introduce a D3-brane to the system. As we have discussed above, it should be supported on either of the two distinguished surfaces in $\mathcal{M}_{p, q}$. Therefore, it creates a surface defect in the four-dimensional theory. In flat spacetime, this defect would preserve half of the supercharges that generate $\mathcal{N}=(0,2)$ supersymmetry on the surface.

The introduction of the surface defect changes the supersymmetric index. One way to determine its effect is to realize the defect as a two-dimensional theory coupled to the four-dimensional theory, and compute the index of the coupled system in the weak coupling limit. It turns out that the defect is represented by a difference operator acting on $\mathrm{SU}(N)$ gauge or flavor fugacities [55]. In the lattice model, the action of this operator is induced by the dashed line inserted by the D3-brane. 


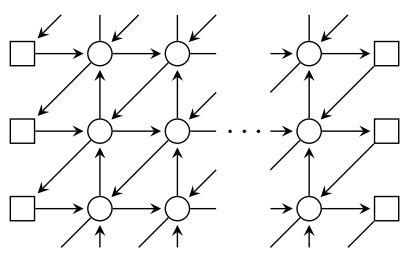

(a)

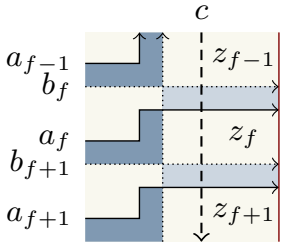

(b)

Figure 7. (a) A quiver describing a class- $\mathcal{S}_{k}$ theory, placed on a finite-length cylinder. (b) A surface defect inserted near the right end of the cylinder.

To be specific, let us take $\Sigma$ to be a finite-length cylinder and consider a lattice that leads to the quiver shown in figure 7(a). The vertical direction is periodic, and there are $k$ nodes in each column. This theory is a $\mathbb{Z}_{k}$ orbifold of an $\mathcal{N}=2$ supersymmetric gauge theory described by a linear quiver, and is a basic example of a class- $\mathcal{S}_{k}$ theory [29]. The supersymmetric index of the theory, or equivalently the partition function of the lattice model, is a function of the dynamical variables associated with the $k \mathrm{SU}(N)$ flavor groups on the right end of the quiver, which we denote by $z_{f}=\left(z_{f, 1}, \ldots, z_{f, N}\right), f=1, \ldots, k$, as well as those associated with the $k \mathrm{SU}(N)$ flavor groups on the left end.

In this theory, we introduce a surface defect that inserts a dashed line in the lattice as in figure $7(\mathrm{~b})$. Let us define an L-operator $\widetilde{L}^{\mathrm{F}}$ by the crossing of a dashed line and a double line in an unshaded background. More explicitly, it has matrix elements

$$
\widetilde{L}^{\mathrm{F}}(a, b)_{i}^{j}=T_{w, j}^{-1} \widetilde{L}^{\mathrm{F}}(a, b ; z, w)_{i}^{j} T_{z, i}^{-1},
$$

with $\widetilde{L}^{\mathrm{F}}(a, b ; z, w)$ given by

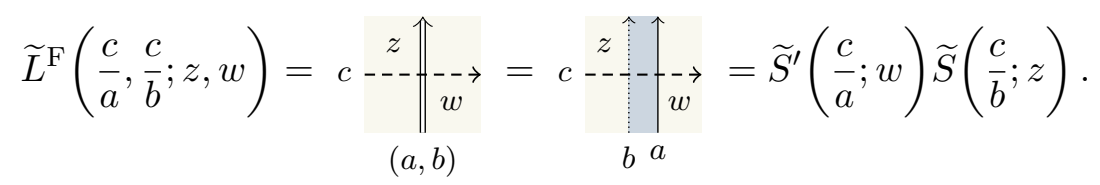

Just as $L^{\mathrm{F}}$ does, this L-operator gives a representation of $E_{\tau, \gamma / 2}\left(\mathfrak{s l}_{N}\right)$. Looking at figure 7(b), we conclude that the surface defect acts on the index as the transfer matrix

$$
\operatorname{Tr}\left(\widetilde{L}_{k}^{\mathrm{F}}\left(\frac{c}{a_{k}}, \frac{c}{b_{k}}\right) \cdots \widetilde{L}_{1}^{\mathrm{F}}\left(\frac{c}{a_{1}}, \frac{c}{b_{1}}\right)\right),
$$

where $\widetilde{L}_{f}^{\mathrm{F}}$ is the copy of $\widetilde{L}^{\mathrm{F}}$ corresponding to the crossing of the dashed line and the $f$ th pair of dotted and solid lines.

A simple calculation yields

$$
\widetilde{L}^{\mathrm{F}}\left(\frac{c}{a}, \frac{c}{b}\right)_{i}^{j}=\frac{\theta\left(d^{N} \sqrt{p / q} a w_{j} / b z_{i}\right)}{\theta\left(d^{N}\right)} \prod_{k(\neq i)} \frac{\theta\left(\sqrt{p / q} a w_{j} / b z_{k}\right)}{\theta\left(z_{k} / z_{i}\right)} T_{z, i}^{-1} T_{w, j}^{-1},
$$

with $d^{-1}=q^{-1 / N} \sqrt{p q} c / b$. For $N=2$, the transfer matrix resulting from this formula reproduces the difference operator obtained in [11], up to an overall factor that is independent of the dynamical variables. For $N>2$, the transfer matrix is consistent with the 
result found in [28], where the action of surface defects in the symmetric powers of the vector representation was computed for restricted values of the spectral parameters.

\subsection{Fusion procedure and exterior powers of the vector representation}

So far we have considered a particular type of surface defect that is created by a single D3-brane ending on the 5-branes. In fact, this is the simplest member of a family of surface defects that can be constructed by similar brane configurations, which in general contain multiple D3-branes and extra NS5-branes. The possible types of these defects are in oneto-one correspondence with the irreducible finite-dimensional representations of $\mathrm{SU}(N)$. What we have discussed above is the case of the vector representation.

Correspondingly, the lattice model should admit a family of dashed lines labeled by the irreducible finite-dimensional representations of $\mathrm{SU}(N)$, not just the one that we have used so far. Indeed, there is a method to generate such a family. This is the fusion procedure, initiated by Kulish, Reshetikhin and Sklyanin [30] in the context of the rational analog of the Belavin model. In [30], the R-matrices for the symmetric and exterior powers of the vector representation were obtained by this procedure. Subsequently, the construction was generalized by Cherednik [31] to arbitrary irreducible representations at the full elliptic level, and applied to the Jimbo-Miwa-Okado model in [56]. ${ }^{6}$

Here we explain the fusion procedure for the exterior powers of the vector representation $V=\mathbb{C}^{N}$, following the treatment in [36]. Concretely, we construct an L-operator $\widetilde{L}_{\wedge^{n} V}^{\mathrm{F}}$ that is associated with the $n$th exterior power $\wedge^{n} V$ and reduces for $n=1$ to the L-operator $\widetilde{L}^{\mathrm{F}}$ defined in (4.1) and (4.2).

Let $J_{n}(V)$ be the subspace of $V^{\otimes n}$ consisting of tensors of the form $\sigma v-\operatorname{sgn}(\sigma) v$, where $\sigma \in S_{n}$ is a permutation of $n$ elements. Then $\wedge^{n} V$ is the quotient $V^{\otimes n} / J_{n}(V)$. We set

$$
\bar{R}^{\mathrm{F}}(u, \lambda)=-\frac{\theta_{1}(u+\gamma)}{2 \theta_{1}(\gamma)} R^{\mathrm{F}}(u, \lambda)
$$

From the definition of $R^{\mathrm{F}}$, it is easy to check $J_{2}(V) \subset \operatorname{ker} \bar{R}^{\mathrm{F}}(-\gamma, \lambda)$. We actually have $\operatorname{ker} \bar{R}^{\mathrm{F}}(-\gamma, \lambda)=J_{2}(V)$ for generic values of $\lambda$, as can be seen from the fact that $\bar{R}^{\mathrm{F}}(-\gamma, \lambda)$ acts on antisymmetric tensors as the identity in the limit $\gamma \rightarrow 0$.

To implement the fusion procedure, we introduce an operator defined by the following diagram:

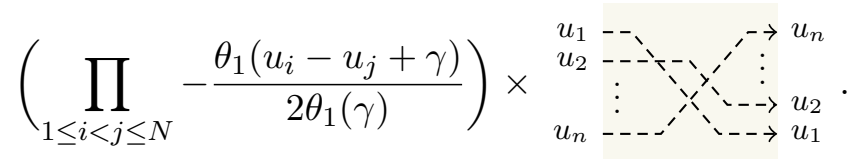

In the middle of the diagram we have a composition of $n(n-1) / 2$ crossings that generate the permutation $(1,2, \ldots, n) \mapsto(n, \ldots, 2,1)$; the details are not important since the YangBaxter equation guarantees that any two such compositions produce the same operator.

\footnotetext{
${ }^{6}$ In connection with surface defects, it should also be noted that the transfer matrices in the symmetric powers of the vector representation are residues of a certain integral operator [29, 57]. In [58], the general fused L-operator were obtained for the elliptic modular double for $N=2$ via reduction of the BSDS R-operator.
} 
The prefactor converts the R-matrices $R_{i j}^{\mathrm{F}}$ that appear in the diagram to $\bar{R}_{i j}^{\mathrm{F}}$. Since any permutation is a product of adjacent transpositions, $J_{n}(V)$ is spanned by tensors of the form $v+P_{i, i+1} v$, with $P_{i, i+1}$ being the swap of the $i$ th and $(i+1)$ th factors. Furthermore, for any adjacent pair $(i, i+1)$, we can find a representation of the above operator such that $\bar{R}_{i, i+1}^{\mathrm{F}}$ appears in the leftmost position in the diagram. It follows that the kernel of this operator, evaluated at $\left(u_{1}, \ldots, u_{n}\right)=(u, u+\gamma, \ldots, u+(n-1) \gamma)$, is generically $J_{n}(V)$.

Now we consider the following tensor product of $n$ copies of $\widetilde{L}^{\mathrm{F}}$ :

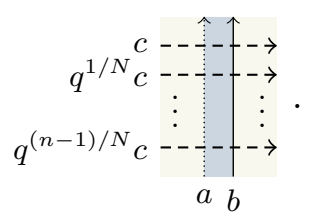

The relation

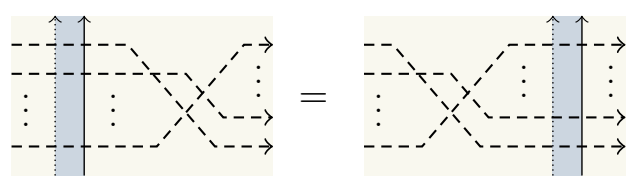

shows that this operator leaves $J_{n}(V)$ invariant. Hence, it descends to a well-defined operator $\widetilde{L}_{\wedge^{n} V}^{\mathrm{F}}(c / a, c / b)$ under the projection $V^{\otimes n} \rightarrow \wedge^{n} V$. This is the desired L-operator.

It is clear that the same argument holds when the pair of vertical lines in (4.8) is replaced with any line that satisfies the Yang-Baxter equation with two dashed lines. Therefore, it makes sense to define a dashed line in the representation $\wedge^{n} V$ as the image of the $n$ copies of dashed lines in the picture under the projection:

$$
\wedge^{n} V, c-\cdots=\left[\begin{array}{r}
q^{c----\rightarrow} \\
q^{1 / N} c---\rightarrow \\
\vdots \\
q^{(n-1) / N} c----\rightarrow
\end{array}\right] .
$$

The fusion procedure just described should reflect the structure of the brane construction of a surface defect in the representation $\wedge^{n} V$. In this construction, we start with $n$ D3-branes ending on a stack of $N$ coincident D5-branes. These D3-branes create $n$ dashed lines, each in the vector representation. To this configuration we add an NS5-brane, and let the other ends of the D3-branes terminate on it; see figure 8. The support of the NS5-brane is chosen in such a way that the D3-branes necessarily become coincident. The exclusion principle for branes then forces the D3-branes to end on separate D5-branes in the stack. There are $N ! / n !(N-n)$ ! different ways in which the D3-branes end on the D5-branes, and they correspond to the standard basis vectors of $\wedge^{n} V$.

In order to check the correspondence between the brane construction and the fusion procedure more quantitatively, let us consider the situation in figure $7(\mathrm{~b})$ again. If we replace the dashed line in the picture with one in the representation $\wedge^{n} V$, then the new line acts on the lattice model as a transfer matrix consisting of $k$ copies of $\widetilde{L}_{\wedge^{n} V}^{\mathrm{F}}$. According to our proposal, it should coincide with the difference operator that represents the action of the corresponding surface defect on the index of the $\mathcal{N}=1$ supersymmetric gauge theory described by the quiver in figure $7(\mathrm{a})$. The gauge theory result is known for $k=1$ [34], so let us calculate the transfer matrix in this case. 


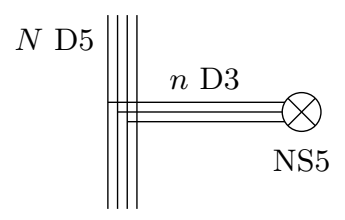

Figure 8. The brane configuration for a surface defect in the representation $\wedge^{n} V$. Here, $(N, n)=$ $(4,3)$ and the dashed line carries the state $e_{1} \wedge e_{2} \wedge e_{3}$

Let $I$ run over the subsets of $\{1, \ldots, N\}$ of order $n$, and $e_{I}=e_{i_{1}} \wedge \cdots \wedge e_{i_{n}}$ be a basis vector of $\wedge^{n} V$, where $i_{r}$ stands for the $r$ th smallest number in $I$. We define

$$
\widetilde{L}_{\wedge^{n} V}^{\mathrm{F}}\left(\frac{c}{a}, \frac{c}{b} ; z, w\right)=\wedge^{n} V, c-\underset{(a, b)}{z \Uparrow} \underset{w}{-\rightarrow}
$$

Then, the transfer matrix we wish to calculate is

$$
\operatorname{Tr} \widetilde{L}_{\wedge^{n} V}^{\mathrm{F}}\left(\frac{c}{a}, \frac{c}{b}\right)=\sum_{I} \widetilde{L}_{\wedge^{n} V}^{\mathrm{F}}\left(\frac{c}{a}, \frac{c}{b} ; z, T_{I}^{-1} z\right)_{I}^{I} T_{I}^{-1}
$$

with $T_{I}=\prod_{i \in I} T_{i}$. The matrix-valued function (4.10) satisfies an RLL relation with a generalization of Felder's R-matrix.

In terms of intertwining operators, the coefficient function in front of $T_{I}^{-1}$ is given by

$$
\begin{aligned}
& \widetilde{L}_{\wedge^{n} V}^{\mathrm{F}}\left(\frac{c}{a}, \frac{c}{b} ; z, T_{I}^{-1} z\right)_{I}^{I} \\
& \quad=\sum_{\sigma \in S_{n}} \operatorname{sgn}(\sigma) \prod_{r=1}^{n}\left[\widetilde{S}^{\prime}\left(q^{(r-1) / N} \frac{c}{a}, T_{\sigma \cdot I_{r}}^{-1} z\right) \widetilde{S}\left(q^{(r-1) / N} \frac{c}{b}, T_{I_{r-1}}^{-1} z\right)\right]_{i_{r}}^{i_{\sigma(r)}},
\end{aligned}
$$

where $I_{r-1}=\left\{i_{1}, \ldots, i_{r-1}\right\}$ and $\sigma \cdot I_{r}=\left\{i_{\sigma(1)}, \ldots, i_{\sigma(r)}\right\}$. Using identity (3.32) and the formula [32]

$$
\begin{gathered}
\sum_{\sigma \in S_{n}} \operatorname{sgn}(\sigma) \prod_{r=1}^{n}\left[\Phi\left(v+(r-1) \gamma, \lambda-\gamma \sum_{s=1}^{r-1} \omega_{i_{s}}\right)^{-1} \Phi\left(u+(r-1) \gamma, \lambda-\gamma \sum_{s=1}^{r-1} \omega_{i_{\sigma(s)}}\right)\right]_{i_{\sigma(r)}}^{i_{r}} \\
=\frac{\theta_{1}(v+(u-v) n / N)}{\theta_{1}(v)} \prod_{\substack{i \in I \\
k \notin I}} \frac{\theta_{1}\left(\lambda_{k i}+(u-v) / N\right)}{\theta_{1}\left(\lambda_{k i}\right)},
\end{gathered}
$$

we can rewrite it as

$$
\widetilde{L}_{\wedge^{n} V}^{\mathrm{F}}\left(\frac{c}{a}, \frac{c}{b} ; z, T_{I}^{-1} z\right)_{I}^{I}=\left(\sqrt{p q} \frac{a}{b}\right)^{n(n-1) / 2} \frac{\theta\left(d^{N}(\sqrt{p / q} a / b)^{n}\right)}{\theta\left(d^{N}\right)} \prod_{\substack{i \in I \\ k \notin I}} \frac{\theta\left(\sqrt{p / q} a z_{i} / b z_{k}\right)}{\theta\left(z_{k} / z_{i}\right)} .
$$

Here $d^{-1}=q^{-1 / N} \sqrt{p q} c / b$ as before. 
Up to a factor independent of the dynamical variable, the transfer matrix computed above reproduces the difference operator that was proposed in [34] to represent the action of the surface defect on the supersymmetric index. (See section 4.1 of [11] for the relation between the spectral parameters used here and the fugacities used in [34].) Varying $n$ from 1 to $N$, we obtain a family of mutually commuting difference operators from the transfer matrix. As was shown in [25], these are related by conjugation by a function to the Ruijsenaars operators [33], the Hamiltonians of the elliptic Ruijsenaars-Schneider model of type $A_{N-1}$.

\section{Acknowledgments}

I would like to thank Kevin Costello for illuminating discussions and directing my attention to Felder's dynamical R-matrix, and Vladimir Bazhanov and Sergey Sergeev for explaining to me the relevance of [23] to their model. I am also grateful to Piotr Sulkowski and Petr Vaško for their comments. This work is supported by the ERC Starting Grant No. 335739 "Quantum fields and knot homologies" funded by the European Research Council under the European Union's Seventh Framework Programme.

Open Access. This article is distributed under the terms of the Creative Commons Attribution License (CC-BY 4.0), which permits any use, distribution and reproduction in any medium, provided the original author(s) and source are credited.

\section{References}

[1] R.J. Baxter, Eight-vertex model in lattice statistics, Phys. Rev. Lett. 26 (1971) 832 [INSPIRE].

[2] R.J. Baxter, Partition function of the eight-vertex lattice model, Annals Phys. 70 (1972) 193 [INSPIRE].

[3] A.A. Belavin, Dynamical symmetry of integrable quantum systems, Nucl. Phys. B 180 (1981) 189 [INSPIRE].

[4] G. Felder, Conformal field theory and integrable systems associated to elliptic curves, in the proceedings of the International Congress of Mathematicians (ICM1994), Zurich, Switzerland (1994), hep-th/9407154 [INSPIRE].

[5] G. Felder, Elliptic quantum groups, talk given at the $11^{\text {th }}$ International Congress of Mathematical Physics, July 18-23, Paris, France (1994), hep-th/9412207 [INSPIRE].

[6] V.V. Bazhanov and S.M. Sergeev, A master solution of the quantum Yang-Baxter equation and classical discrete integrable equations, Adv. Theor. Math. Phys. 16 (2012) 65 [arXiv: 1006.0651] [INSPIRE].

[7] V.V. Bazhanov and S.M. Sergeev, Elliptic gamma-function and multi-spin solutions of the Yang-Baxter equation, Nucl. Phys. B 856 (2012) 475 [arXiv:1106.5874] [inSPIRE].

[8] S.E. Derkachov and V.P. Spiridonov, Yang-Baxter equation, parameter permutations and the elliptic beta integral, Russ. Math. Surveys 68 (2013) 1027 [arXiv:1205.3520] [INSPIRE].

[9] R.J. Baxter, Eight-vertex model in lattice statistics and one-dimensional anisotropic Heisenberg chain. 2. Equivalence to a generalized ice-type lattice model, Annals Phys. 76 (1973) 25 [INSPIRE]. 
[10] M. Jimbo, T. Miwa and M. Okado, Solvable lattice models whose states are dominant integral weights of $A_{n-1}^{(1)}$, Lett. Math. Phys. 14 (1987) 123.

[11] K. Maruyoshi and J. Yagi, Surface defects as transfer matrices, PTEP 2016 (2016) 113B01 [arXiv: 1606.01041] [INSPIRE].

[12] V.P. Spiridonov, Elliptic beta integrals and solvable models of statistical mechanics, Contemp. Math. 563 (2012) 181 [arXiv: 1011.3798] [INSPIRE].

[13] M. Yamazaki, Quivers, YBE and 3-manifolds, JHEP 05 (2012) 147 [arXiv:1203.5784] [INSPIRE].

[14] J. Yagi, Quiver gauge theories and integrable lattice models, JHEP 10 (2015) 065 [arXiv: 1504.04055] [INSPIRE].

[15] J. Yagi, Branes and integrable lattice models, Mod. Phys. Lett. A 32 (2016) 1730003 [arXiv: 1610.05584] [INSPIRE].

[16] A. Hanany and K.D. Kennaway, Dimer models and toric diagrams, hep-th/0503149 [INSPIRE].

[17] S. Franco, A. Hanany, K.D. Kennaway, D. Vegh and B. Wecht, Brane dimers and quiver gauge theories, JHEP 01 (2006) 096 [hep-th/0504110] [INSPIRE].

[18] N. Seiberg, Electric-magnetic duality in supersymmetric non-Abelian gauge theories, Nucl. Phys. B 435 (1995) 129 [hep-th/9411149] [INSPIRE].

[19] K. Costello, Supersymmetric gauge theory and the Yangian, arXiv:1303.2632 [INSPIRE].

[20] K. Costello, Integrable lattice models from four-dimensional field theories, Proc. Symp. Pure Math. 88 (2014) 3 [arXiv: 1308.0370] [INSPIRE].

[21] E.K. Sklyanin, Some algebraic structures connected with the Yang-Baxter equation. Representations of quantum algebras, Funct. Anal. Appl. 17 (1983) 273 [INSPIRE].

[22] V.V. Bazhanov and Yu. G. Stroganov, Chiral Potts model as a descendant of the six vertex model, J. Statist. Phys. 59 (1990) 799 [INSPIRE].

[23] S.M. Sergeev, $\mathbb{Z}_{N}^{n-1}$ broken model, IHEP preprint 92-7 (1992).

[24] Y.-h. Quano and A. Fujii, Yang-Baxter equation for broken $Z^{\otimes n-1}$ models, Mod. Phys. Lett. A 8 (1993) 1585 [hep-th/9210063] [INSPIRE].

[25] K. Hasegawa, On the crossing symmetry of the broken $Z_{N}$-symmetric solution of the Yang-Baxter equation, in Representation theory of Lie groups and Lie algebras, T. Kawazoe et al. eds., World Scientific, River Edge, U.S.A. (1992).

[26] M. Jimbo, T. Miwa and M. Okado, Local state probabilities of solvable lattice models: an $A_{n}^{(1)}-1$ family, Nucl. Phys. B 300 (1988) 74 [INSPIRE].

[27] M. Jimbo, T. Miwa and M. Okado, Solvable lattice models related to the vector representation of classical simple lie algebras, Commun. Math. Phys. 116 (1988) 507 [InSPIRE].

[28] Y. Ito and Y. Yoshida, Superconformal index with surface defects for class $\mathcal{S}_{k}$, arXiv: 1606.01653 [INSPIRE].

[29] D. Gaiotto and S.S. Razamat, $\mathcal{N}=1$ theories of class $\mathcal{S}_{k}$, JHEP 07 (2015) 073 [arXiv: 1503.05159] [INSPIRE].

[30] P.P. Kulish, N. Yu. Reshetikhin and E.K. Sklyanin, Yang-Baxter equation and representation theory. 1., Lett. Math. Phys. 5 (1981) 393 [inSPIRE]. 
[31] I.V. Cherednik, On "quantum" deformations of irreducible finite-dimensional representations of $\mathfrak{g l}_{N}$, Sov. Math. Dokl. 33 (1986) 507 [Dokl. Akad. Nauk CCCP 33 (1986) 1076].

[32] K. Hasegawa, Ruijsenaars' commuting difference operators as commuting transfer matrices, Commun. Math. Phys. 187 (1997) 289.

[33] S.N.M. Ruijsenaars, Complete integrability of relativistic Calogero-Moser systems and elliptic function identities, Commun. Math. Phys. 110 (1987) 191 [INSPIRE].

[34] M. Bullimore, M. Fluder, L. Hollands and P. Richmond, The superconformal index and an elliptic algebra of surface defects, JHEP 10 (2014) 062 [arXiv:1401.3379] [INSPIRE].

[35] M.P. Richey and C.A. Tracy, $\mathbb{Z}_{n}$ Baxter model: symmetries and the Belavin parametrization, J. Stat. Phys. 42 (1986) 311.

[36] G. Felder and A. Varchenko, Elliptic quantum groups and Ruijsenaars models, J. Stat. Phys. 89 (1997) 963 [q-alg/9704005].

[37] V.P. Spiridonov, A Bailey tree for integrals, Theor. Math. Phys. 139 (2004) 536 [Teoret. Mat. Fiz. 139 (2004) 104] [math/0312502].

[38] V.P. Spiridonov and S.O. Warnaar, Inversions of integral operators and elliptic beta integrals on root systems, Adv. Math. 207 (2006) 91 [math/0411044].

[39] V.P. Spiridonov, On the elliptic beta function, Russ. Math. Surv. 56 (2001) 185 [Uspekhi Mat. Nauk 56 (2001) 181].

[40] V.P. Spiridonov, Theta hypergeometric integrals, St. Petersburg Math. J. 15 (2004) 929 [Algebra i Analiz 15 (2003) 161] [math/0303205].

[41] E.M. Rains, Transformations of elliptic hypergeometric integrals, Ann. Math 171 (2010) 169 [math/0309252].

[42] V.V. Bazhanov, R.M. Kashaev, V.V. Mangazeev and Yu.G. Stroganov, $\left(Z_{N} \times\right)^{n-1}$ generalization of the chiral Potts model, Commun. Math. Phys. 138 (1991) 393 [InSPIRE].

[43] P. Etingof and A. Varchenko, Solutions of the quantum dynamical Yang-Baxter equation and dynamical quantum groups, Commun. Math. Phys. 196 (1998) 591 [q-alg/9708015].

[44] E. Koelink, Y. van Norden and H. Rosengren, Elliptic U(2) quantum group and elliptic hypergeometric series, Commun. Math. Phys. 245 (2004) 519 [math/0304189].

[45] J. T. Hartwig, The elliptic GL(n) dynamical quantum group as an $\mathfrak{h}$-Hopf algebroid, Int. J. Math. Math. Sci. (2009) 545892 [arXiv:0803.3815].

[46] C. Romelsberger, Counting chiral primaries in $N=1, D=4$ superconformal field theories, Nucl. Phys. B 747 (2006) 329 [hep-th/0510060] [InSPIRE].

[47] J. Kinney, J.M. Maldacena, S. Minwalla and S. Raju, An index for 4 dimensional super conformal theories, Commun. Math. Phys. 275 (2007) 209 [hep-th/0510251] [INSPIRE].

[48] G. Festuccia and N. Seiberg, Rigid supersymmetric theories in curved superspace, JHEP 06 (2011) 114 [arXiv:1105.0689] [INSPIRE].

[49] C. Closset, T.T. Dumitrescu, G. Festuccia and Z. Komargodski, The geometry of supersymmetric partition functions, JHEP 01 (2014) 124 [arXiv:1309.5876] [INSPIRE].

[50] F. Nieri and S. Pasquetti, Factorisation and holomorphic blocks in 4d, JHEP 11 (2015) 155 [arXiv: 1507.00261] [INSPIRE].

[51] F. Nieri, An elliptic Virasoro symmetry in 6d, arXiv:1511.00574 [INSPIRE]. 
[52] V.P. Spiridonov, Continuous biorthogonality of the elliptic hypergeometric function, St. Petersburg Math. J. 20 (2009) 791 [Alg. i Anal. 20 (2008) 155] [arXiv:0801.4137].

[53] L. Faddeev, Modular double of a quantum group, in Conférence Moshé Flato 1999, Vol. I (Dijon), Kluwer Acad. Publ., Dordrecht The Netherlands (2000).

[54] A. Hanany and D. Vegh, Quivers, tilings, branes and rhombi, JHEP 10 (2007) 029 [hep-th/0511063] [INSPIRE].

[55] A. Gadde and S. Gukov, 2d index and surface operators, JHEP 03 (2014) 080 [arXiv: 1305.0266] [INSPIRE].

[56] M. Jimbo, A. Kuniba, T. Miwa and M. Okado, The $A_{n}^{(1)}$ face models, Commun. Math. Phys. 119 (1988) 543 [INSPIRE].

[57] D. Gaiotto, L. Rastelli and S.S. Razamat, Bootstrapping the superconformal index with surface defects, JHEP 01 (2013) 022 [arXiv:1207.3577] [INSPIRE].

[58] D. Chicherin, S.E. Derkachov and V.P. Spiridonov, New elliptic solutions of the Yang-Baxter equation, Commun. Math. Phys. 345 (2016) 507 [arXiv:1412.3383] [InSPIRE]. 